BULLETIN (New Series) OF THE

AMERICAN MATHEMATICAL SOCIETY

Volume 49, Number 3, July 2012, Pages 415-442

S 0273-0979(2011)01368-4

Article electronically published on December 28, 2011

\title{
UNIQUENESS PROPERTIES OF SOLUTIONS TO SCHRÖDINGER EQUATIONS
}

\author{
L. ESCAURIAZA, C. E. KENIG, G. PONCE, AND L. VEGA
}

\section{INTRODUCTION}

To place the subject of this paper in perspective, we start out with a brief discussion of unique continuation. Consider solutions to

$$
\Delta u(x)=\sum_{j=1}^{n} \frac{\partial^{2} u}{\partial x_{j}^{2}}(x)=0
$$

(harmonic functions) in the unit ball $\left\{x \in \mathbb{R}^{n}:|x|<1\right\}$. When $n=2$, these functions are real parts of holomorphic functions, and so, if they vanish of infinite order at $x=0$, they must vanish identically. We call this the strong unique continuation property (SUCP). The same result holds for $n>2$, since harmonic functions are still real analytic in $\left\{x \in \mathbb{R}^{n}:|x|<1\right\}$. In fact, it is well known that if $P(x, D)$ is a linear elliptic differential operator with real analytic coefficients and $P(x, D) u=0$ in a open set $\Omega \subset \mathbb{R}^{n}$, then $u$ is real analytic in $\Omega$. Hence, the SUCP also holds for such solutions. Through the work of Hadamard [27] on the uniqueness of the Cauchy problem (which is closely related to the SUCP) it became clear (for applications in nonlinear problems) that it would be desirable to establish the SUCP for operators whose coefficients are not necessarily real analytic, or even $C^{\infty}$. The first results in this direction were found in the pioneering work of Carleman 9] (when $n=2$ ) and Müller [47] (when $n>2$ ), who proved the SUCP for

$$
P(x, D)=\Delta+V(x), \quad \text { with } \quad V \in L_{\mathrm{loc}}^{\infty}\left(\mathbb{R}^{n}\right) .
$$

In order to establish his result, Carleman introduced a method (the method of Carleman estimates) which has permeated the subject ever since. In this context, an example of a Carleman estimate is:

$$
\begin{aligned}
& \text { For } f \in C_{0}^{\infty}\left(\left\{x \in \mathbb{R}^{n}:|x|<1\right\}-\{0\}\right), \alpha>0 \text { and } \\
& w(r)=r \exp \left(\int_{0}^{r} \frac{e^{-s}-1}{s} d s\right),
\end{aligned}
$$

one has

$$
\alpha^{3} \int w^{-1-2 \alpha}(|x|) f^{2}(x) d x \leq c \int w^{2-2 \alpha}(|x|)|\Delta f(x)|^{2} d x,
$$

with $c$ independent of $\alpha$.

Received by the editors September 16, 2011.

2010 Mathematics Subject Classification. Primary 35Q55.

Key words and phrases. Schrödinger evolutions.

The first and fourth authors are supported by MEC grant, MTM2004-03029, the second and third authors by NSF grants DMS-0968472 and DMS-0800967, respectively. 
For a proof of this estimate, see [25], 7]. The SUCP of Carleman-Müller follows easily from (1.2) (see 37] for instance).

In the late 1950s and 1960s there was a great deal of activity on the subject of SUCP and the closely related uniqueness in the Cauchy problem, some highlights being [1] and [8], respectively, both of which use the method of Carleman estimates. These results and methods have had a multitude of applications to many areas of analysis, including to nonlinear problems. (For a recent example, see 38] for an application to energy critical nonlinear wave equations).

In connection with the Carleman-Müller SUCP a natural question is, How fast is a solution $u$ allowed to vanish before it must vanish identically?

By considering $n=2, u\left(x_{1}, x_{2}\right)=\Re\left(x_{1}+i x_{2}\right)^{N}$, we see that to make sense of the question, a normalization is required; for instance,

$$
\sup _{|x|<3 / 4}|u(x)| \geq 1, \quad\|u\|_{L^{\infty}(|x|<1)}<\infty .
$$

We refer to questions of this type as quantitative unique continuation. It is also of interest to consider unique continuation type questions around the point at infinity. For instance, a conjecture of E. M. Landis [41] states if

$$
\Delta u+V u=0, \quad x \in \mathbb{R}^{n}, \quad \text { with }\|V\|_{\infty} \leq 1, \quad\|u\|_{\infty}<\infty,
$$

and for some $\epsilon>0$ one has

$$
|u(x)| \leq c_{\epsilon} e^{-c_{\epsilon}|x|^{1+\epsilon}},
$$

then $u \equiv 0$.

For the case of complex valued potentials $V(x)$, this conjecture was disproved by Meshkov [45] who constructed $V, u, u \not \equiv 0$ with

$$
|u(x)| \leq c e^{-c|x|^{4 / 3}}, \quad n \geq 2 .
$$

Meshkov also showed that if

$$
|u(x)| \leq c_{\epsilon} e^{-c_{\epsilon}|x|^{4 / 3+\epsilon}}, \quad \text { for some } \quad \epsilon>0,
$$

then $u \equiv 0$.

It turns out that a quantitative formulation of this can also be proved, as was done in [7, and this was crucial for the resolution in [7] of a long-standing problem in disordered media, namely Anderson localization near the bottom of the spectrum for the continuous Anderson-Bernoulli model in $\mathbb{R}^{n}, n \geq 1$.

Next, we turn to versions of unique continuation for evolution equations. We start with parabolic equations and consider solutions of

$$
\partial_{t} u-\Delta u=W \cdot \nabla u+V u, \quad \text { with } \quad\|W\|_{\infty}+\|V\|_{\infty}<\infty
$$

(or equivalently $\left|\partial_{t} u-\Delta u\right| \leq M(|\nabla u|+|u|)$ ). Using a parabolic analog of the Carleman estimate described earlier, one can show that if

$$
\left|\partial_{t} u-\Delta u\right| \leq M(|\nabla u|+|u|), \quad(x, t) \in\left\{x \in \mathbb{R}^{n}:|x|<4 R\right\} \times\left[t_{0}, t_{1}\right], \quad R>0,
$$

with $|u(x)| \leq A$ and

$$
u \equiv 0, \quad(x, t) \in\left\{x \in \mathbb{R}^{n}: R<|x|<4 R\right\} \times\left[t_{0}, t_{1}\right],
$$

then

$$
u \equiv 0, \quad(x, t) \in\left\{x \in \mathbb{R}^{n}:|x|<R\right\} \times\left[t_{0}, t_{1}\right] .
$$

We call this type of result unique continuation through spatial boundaries (see [25], [55], and references therein for this type of result and strengthenings of it). 
This result is closely related to the elliptic SUCP discussed before. On the other hand, for parabolic equations, there is also a backward uniqueness principle, which is very useful in applications to control theory (see [44] for an early result in this direction): Consider solutions to

$$
\left|\partial_{t} u-\Delta u\right| \leq M(|\nabla u|+|u|), \quad(x, t) \in \mathbb{R}^{n} \times(0,1],
$$

with $\|u\|_{\infty} \leq A$. Then, if $u(\cdot, 1) \equiv 0$, we must have $u \equiv 0$. This result is also proved through Carleman estimates (see [4]).

Recently, a strengthening of this result has been obtained in 24], where one considers solutions only defined in $R_{+}^{n} \times(0,1], R_{+}^{n}=\left\{\left(x_{1}, \ldots, x_{n}\right) \in \mathbb{R}^{n}: x_{1}>0\right\}$, without any assumptions on $u$ at $x_{1}=0$, and still obtains the backward uniqueness result. This strengthening had an important application to nonlinear equations, allowing the authors of 24] to establish a long-standing conjecture of J. Leray on regularity and uniqueness of solutions to the Navier-Stokes equations (see also [52] for a recent extension).

Finally, we turn to dispersive equations. Typical examples of these are the $k$ generalized $\mathrm{KdV}$ equation

$$
\partial_{t} u+\partial_{x}^{3} u+u^{k} \partial_{x} u=0, \quad(x, t) \in \mathbb{R} \times \mathbb{R}, \quad k \in \mathbb{Z}^{+},
$$

and the nonlinear Schrödinger equation

$$
\partial_{t} u=i\left(\Delta u \pm|u|^{p-1} u\right), \quad(x, t) \in \mathbb{R}^{n} \times \mathbb{R}, \quad p>1 .
$$

These equations model phenomena of wave propagation and have been extensively studied in the last 30 years or so.

For these equations, unique continuation through spatial boundaries also holds, as was shown by Saut-Scheurer [51] for the KdV-type equations and by Izakov 35] for Schrödinger-type equations. (All of these results were established through Carleman estimates.) These equations however are time reversible (no preferred time direction) and so backward uniqueness is immediate, unlike in parabolic problems. Once more in connection with control theory, this time for dispersive equations, Zhang [56] showed, for solutions of

$$
\partial_{t} u=i\left(\partial_{x}^{2} u \pm|u|^{2} u\right), \quad(x, t) \in \mathbb{R} \times[0,1],
$$

that if $u(x, t)=0$ for $(x, t) \in(-\infty, a) \times\{0,1\}($ or $(x, t) \in(a, \infty) \times\{0,1\})$ for some $a \in \mathbb{R}$, then $u \equiv 0$. Zhang's proof was based on the inverse scattering method, which uses that this is a completely integrable model, and did not apply to other nonlinearities or dimensions. This type of result was extended to the $k$-generalized $\mathrm{KdV}$ equation in (1.3) and the general nonlinear Schrödinger equation in (1.4) in all dimensions (where inverse scattering is no longer available) using suitable Carleman estimates (see [39, 33], 34, and references therein).

For recent surveys of the results presented so far, see [36, 37.

Returning to backward uniqueness for parabolic equations, in analogy with Landis's elliptic conjecture mentioned earlier, Landis and Oleinik [43] conjectured that in the backward uniqueness result one can replace the hypothesis $u(\cdot, 1) \equiv 0$ with the weaker one

$$
|u(x, 1)| \leq c_{\epsilon} e^{-c_{\epsilon}|x|^{2+\epsilon}}, \text { for some } \epsilon>0 .
$$

This is indeed true and was established in [18] and [49. Similarly, one can conjecture (as was done in [20]) that for Schrödinger equations, if

$$
|u(x, 0)|+|u(x, 1)| \leq c_{\epsilon} e^{-c_{\epsilon}|x|^{2+\epsilon}}, \text { for some } \epsilon>0,
$$


then $u \equiv 0$. This was established in [18].

In analogy with the improvement of backward uniqueness in 24, one can show that it suffices to deal with solutions in $\mathbb{R}_{+}^{n} \times(0,1]$ (for parabolic problems) and require

$$
|u(x, 1)| \leq c_{\epsilon} e^{-c_{\epsilon} x_{1}^{2+\epsilon}}, \quad x_{1}>0, \quad \text { for some } \quad \epsilon>0,
$$

to conclude that $u \equiv 0$ (49]), and that for the Schrödinger equations it suffices to have $u$ a solution in $\mathbb{R}_{+}^{n} \times[0,1]$, with

$$
|u(x, 0)|+|u(x, 1)| \leq c_{\epsilon} e^{-c_{\epsilon} x_{1}^{2+\epsilon}}, \quad x_{1}>0, \quad \text { for some } \quad \epsilon>0,
$$

to conclude that $u \equiv 0$, as we will prove in section 5 of this paper.

In [16] it was pointed out for the first time (see also [10]) that both the results in [18] and in [16] in the case of the free heat equation,

$$
\partial_{t} u=\Delta u
$$

and the free Schrödinger equation,

$$
\partial_{t} u=i \Delta u
$$

respectively, are in fact a corollary of the more precise Hardy uncertainty principle for the Fourier transform, which says,

$$
\begin{aligned}
& \text { if } f(x)=O\left(e^{-|x|^{2} / \beta^{2}}\right), \widehat{f}(\xi)=O\left(e^{-4|\xi|^{2} / \alpha^{2}}\right) \text { and } 1 / \alpha \beta>1 / 4 \text {, then } \\
& f \equiv 0 \text {, and if } 1 / \alpha \beta=1 / 4, f(x)=c e^{-|x|^{2} / \beta^{2}} \text {, as will be discussed } \\
& \text { below. }
\end{aligned}
$$

Thus, in a series of papers $([16]-[23$, [11]) we took up the task of finding the sharp version of the Hardy uncertainty principle, in the context of evolution equations. The results obtained have already yielded new results on nonlinear equations. For instance in 21] and 23] we have found applications to the decay of concentration profiles of possible self-similar type blow-up solutions of nonlinear Schrödnger equations and to the decay of possible solitary wave type solutions of nonlinear Schrödinger equations.

In the rest of this work we shall review some of our recent results concerning unique continuation properties of solutions of Schrödinger equations of the form

$$
\partial_{t} u=i(\Delta u+F(x, t, u, \bar{u})), \quad(x, t) \in \mathbb{R}^{n} \times \mathbb{R} .
$$

We shall be mainly interested in the case where

$$
F(x, t, u, \bar{u})=V(x, t) u(x, t)
$$

describes the evolution of the Schrödinger flow with a time-dependent potential $V(x, t)$, and in the semilinear case

$$
F(x, t, u, \bar{u})=F(u, \bar{u})
$$

with $F: \mathbb{C} \times \mathbb{C} \rightarrow \mathbb{C}, F(0,0)=\partial_{u} F(0,0)=\partial_{\bar{u}} F(0,0)=0$.

Let us consider a familiar dispersive model, the $k$-generalized Korteweg-de Vries equation (1.3), and recall a theorem established in [17]: 
Theorem 1. There exists $c_{0}>0$ such that for any pair

$$
u_{1}, u_{2} \in C\left([0,1]: H^{4}(R) \cap L^{2}\left(|x|^{2} d x\right)\right)
$$

of solutions of (1.3), if

$$
u_{1}(\cdot, 0)-u_{2}(\cdot, 0), \quad u_{1}(\cdot, 1)-u_{2}(\cdot, 1) \in L^{2}\left(e^{c_{0} x_{+}^{3 / 2}} d x\right),
$$

then $u_{1} \equiv u_{2}$.

Above we have used the notation $x_{+}=\max \{x ; 0\}$.

Notice that by taking $u_{2} \equiv 0$ Theorem 1 gives a restriction on the possible decay of a nontrivial solution of (1.3) at two different times. The power $3 / 2$ in the exponent in (1.9) reflects the asymptotic behavior of the Airy function. More precisely, the solution of the initial value problem (IVP)

$$
\left\{\begin{array}{l}
\partial_{t} v+\partial_{x}^{3} v=0 \\
v(x, 0)=v_{0}(x)
\end{array}\right.
$$

is given by the group $\{U(t): t \in R\}$

$$
U(t) v_{0}(x)=\frac{1}{\sqrt[3]{3 t}} A i\left(\frac{\cdot}{\sqrt[3]{3 t}}\right) * v_{0}(x)
$$

where

$$
A i(x)=c \int_{-\infty}^{\infty} e^{i x \xi+i \xi^{3}} d \xi
$$

is the Airy function which satisfies the estimate

$$
|A i(x)| \leq c\left(1+x_{-}\right)^{-1 / 4} e^{-c x_{+}^{3 / 2}} .
$$

It was also shown in [17] that Theorem 1 is optimal:

Theorem 2. There exists $u_{0} \in S(\mathbb{R}), u_{0} \not \equiv 0$ and $\Delta T>0$ such that the IVP associated to the $k-g K d V$ equation (1.3) with data $u_{0}$ has solution

$$
u \in C([0, \Delta T]: \mathbb{S}(\mathbb{R})),
$$

satisfying

for some constant $\tilde{d}>0$.

$$
|u(x, t)| \leq \tilde{d} e^{-x^{3 / 2} / 3}, \quad x>1, \quad t \in[0, \Delta T],
$$

In the case of the free Schrödinger group $\left\{e^{i t \Delta}: t \in \mathbb{R}\right\}$

$$
e^{i t \Delta} u_{0}(x)=\left(e^{-i|\xi|^{2} t} \widehat{u}_{0}\right)^{\vee}(x)=\frac{e^{i|\cdot|^{2} / 4 t}}{(4 \pi i t)^{n / 2}} * u_{0}(x),
$$

the fundamental solution does not decay. However, one has the identity

$$
\begin{aligned}
u(x, t) & =e^{i t \Delta} u_{0}(x)=\int_{\mathbb{R}^{n}} \frac{e^{i|x-y|^{2} / 4 t}}{(4 \pi i t)^{n / 2}} u_{0}(y) d y \\
& =\frac{e^{i|x|^{2} / 4 t}}{(4 \pi i t)^{n / 2}} \int_{\mathbb{R}^{n}} e^{-2 i x \cdot y / 4 t} e^{i|y|^{2} / 4 t} u_{0}(y) d y \\
& =\frac{e^{i|x|^{2} / 4 t}}{(2 i t)^{n / 2}}\left(e^{i \widehat{|\cdot|^{2} / 4 t}} u_{0}\right)\left(\frac{x}{2 t}\right),
\end{aligned}
$$


where

$$
\widehat{f}(\xi)=(2 \pi)^{-n / 2} \int_{\mathbb{R}^{n}} e^{-i \xi \cdot x} f(x) d x
$$

Hence,

$$
c_{t} e^{-i|x|^{2} / 4 t} u(x, t)=\left(e^{i \widehat{||^{2} / 4 t}} u_{0}\right)\left(\frac{x}{2 t}\right), \quad c_{t}=(2 i t)^{n / 2},
$$

which tells us that $e^{-i|x|^{2} / 4 t} u(x, t)$ is a multiple of the rescaled Fourier transform of $e^{i|y|^{2} / 4 t} u_{0}(y)$. Thus, as we pointed out earlier, the behavior of the solution of the free Schrödinger equation is closely related to uncertainty principles for the Fourier transform. We shall study these uncertainty principles and their relation with the uniqueness properties of the solution of the Schrödinger equation (1.6). In the early 1930s N. Wiener's remark (see [28, [32, and [46]):

"a pair of transforms $f$ and $g(\widehat{f})$ cannot both be very small",

motivated the works of G. H. Hardy [28, G. W. Morgan [46], and A. E. Ingham [32, which will be considered in detail in this note. However, before that we shall return to a review of some previous results concerning uniqueness properties of solutions of the Schrödinger equation which we mentioned earlier and which were not motivated by the formula (1.11).

For solutions $u(x, t)$ of the 1 -D cubic Schrödinger equation (1.5) B. Y. Zhang [56] showed,

$$
\begin{aligned}
& \text { If } u(x, t)=0 \text { for }(x, t) \in(-\infty, a) \times\{0,1\}(\text { or }(x, t) \in(a, \infty) \times \\
& \{0,1\}) \text { for some } a \in \mathbb{R} \text {, then } u \equiv 0 \text {. }
\end{aligned}
$$

As was mentioned before, his proof is based on the inverse scattering method, which uses the fact that the equation in (1.5) is a completely integrable model.

In 39] it was proved under general assumptions on $F$ in (1.8) that:

If $u_{1}, u_{2} \in C\left([0,1]: H^{s}\left(\mathbb{R}^{n}\right)\right)$, with $s>\max \{n / 2 ; 2\}$ are solutions of the equation (1.6) with $F$ as in (1.8) such that

$$
u_{1}(x, t)=u_{2}(x, t), \quad(x, t) \in \Gamma_{x_{0}}^{c} \times\{0,1\},
$$

where $\Gamma_{x_{0}}^{c}$ denotes the complement of a cone $\Gamma_{x_{0}}$ with vertex $x_{0} \in$ $\mathbb{R}^{n}$ and opening $<180^{\circ}$, then $u_{1} \equiv u_{2}$.

(For further results in this direction see [39, 33], 34], and references therein).

A key step in the proof in [39] was the following uniform exponential decay estimate:

Lemma 1. There exists $\epsilon_{0}>0$ such that if

$$
\mathbb{V}: \mathbb{R}^{n} \times[0,1] \rightarrow \mathbb{C}, \quad \text { with }\|\mathbb{V}\|_{L_{t}^{1} L_{x}^{\infty}} \leq \epsilon_{0},
$$

and $u \in C\left([0,1]: L^{2}\left(\mathbb{R}^{n}\right)\right)$ is a strong solution of the IVP

$$
\left\{\begin{array}{l}
\partial_{t} u=i(\Delta+\mathbb{V}(x, t)) u+\mathbb{G}(x, t), \\
u(x, 0)=u_{0}(x),
\end{array}\right.
$$

with

$$
u_{0}, u_{1} \equiv u(\cdot, 1) \in L^{2}\left(e^{2 \lambda \cdot x} d x\right), \mathbb{G} \in L^{1}\left([0,1]: L^{2}\left(e^{2 \lambda \cdot x} d x\right)\right),
$$


for some $\lambda \in \mathbb{R}^{n}$, then there exists $c_{n}$ independent of $\lambda$ such that

$$
\begin{aligned}
& \sup _{0 \leq t \leq 1}\left\|e^{\lambda \cdot x} u(\cdot, t)\right\|_{L^{2}\left(\mathbb{R}^{n}\right)} \\
& \leq c_{n}\left(\left\|e^{\lambda \cdot x} u_{0}\right\|_{L^{2}\left(\mathbb{R}^{n}\right)}+\left\|e^{\lambda \cdot x} u_{1}\right\|_{L^{2}\left(\mathbb{R}^{n}\right)}+\int_{0}^{1}\left\|e^{\lambda \cdot x} \mathbb{G}(\cdot, t)\right\|_{L^{2}\left(\mathbb{R}^{n}\right)} d t\right) .
\end{aligned}
$$

Notice that in the above result one assumes the existence of a reference $L^{2}$ solution $u$ of the equation (1.13) and then under the hypotheses (1.12) and (1.14) shows that the exponential decay in the time interval $[0,1]$ is preserved.

The estimate (1.15) can be combined with the subordination formula

$$
e^{\gamma|x|^{p} / p} \simeq \int_{\mathbb{R}^{n}} e^{\gamma^{1 / p} \lambda \cdot x-|\lambda|^{q} / q}|\lambda|^{n(q-2) / 2} d \lambda, \quad \forall x \in \mathbb{R}^{n} \text { and } p>1,
$$

to get that for any $\alpha>0$ and $a>1$

$$
\begin{aligned}
& \sup _{0 \leq t \leq 1}\left\|e^{\alpha|x|^{a}} u(\cdot, t)\right\|_{L^{2}\left(\mathbb{R}^{n}\right)} \\
& \quad \leq c_{n}\left(\left\|e^{\alpha|x|^{a}} u_{0}\right\|_{L^{2}\left(\mathbb{R}^{n}\right)}+\left\|e^{\alpha|x|^{a}} u_{1}\right\|_{L^{2}\left(\mathbb{R}^{n}\right)}+\int_{0}^{1}\left\|e^{\alpha|x|^{a}} \mathbb{G}(\cdot, t)\right\|_{L^{2}\left(\mathbb{R}^{n}\right)} d t\right) .
\end{aligned}
$$

Under appropriate assumptions on the potential $V(x, t)$ in (1.7), one writes

$$
V(x, t) u=\chi_{R} V(x, t) u+\left(1-\chi_{R}\right) V(x, t) u=\mathbb{V}(x, t) u+\mathbb{G}(x, t),
$$

with $\chi_{R} \in C_{0}^{\infty}, \chi_{R}(x)=1,|x|<R$, supported in $|x|<2 R$, and applies the estimate (1.17) by fixing $R$ sufficiently large. Also under appropriate hypothesis on $F$ and $u$, a similar argument can be used for the semilinear equation in (1.8).

The estimate (1.17) gives a control on the decay of the solution in the whole time interval in terms of that at the endpoints and that of the external force. As we shall see below, a key idea will be to get improvements of this estimate based on logarithmically convex versions of it.

We recall that if one considers the equation (1.6) with initial data $u_{0} \in \mathbb{S}\left(\mathbb{R}^{n}\right)$ and a smooth potential $V(x, t)$ in (1.7) or smooth nonlinearity $F$ in (1.8), it follows that the corresponding solution satisfies that $u \in C\left([-T, T]: \mathbb{S}\left(\mathbb{R}^{n}\right)\right)$. This can be proved using the commutative property of the operators

$$
L=\partial_{t}-i \Delta, \quad \text { and } \quad \Gamma_{j}=x_{j}+2 t \partial_{x_{j}}, j=1, \ldots, n ;
$$

see 29]-30. From the proof of this fact one also has that the persistence property of the solution $u=u(x, t)$ (i.e., if the data $u_{0} \in X$, a function space, then the corresponding solution $u(\cdot)$ describes a continuous curve in $X, u \in C([-T, T]: X)$, $T>0)$ with data $u_{0} \in L^{2}\left(|x|^{m}\right)$ can only hold if $u_{0} \in H^{s}\left(\mathbb{R}^{n}\right)$ with $s \geq 2 m$. Roughly speaking, for exponential weights one has a more involved argument where the time direction plays a role. Considering the IVP for the one-dimensional free Schrödinger equation

$$
\left\{\begin{array}{l}
\partial_{t} u=i \partial_{x}^{2} u, \quad x, t \in \mathbb{R}, \\
u(x, 0)=u_{0}(x) \in L^{2}(\mathbb{R}),
\end{array}\right.
$$

and assuming that $e^{\beta x} u_{0} \in L^{2}(\mathbb{R}), \beta>0$, then one formally has that

$$
v(x, t)=e^{\beta x} u(x, t)
$$

satisfies the equation

$$
\partial_{t} v=i\left(\partial_{x}-\beta\right)^{2} v
$$


Thus,

$$
v(x, \pm 1)=e^{\beta x} u(x, \pm 1) \in L^{2}(\mathbb{R}) \quad \text { if } \quad e^{ \pm 2 \beta \xi} \widehat{e^{\beta x} u_{0}} \in L^{2}(\mathbb{R}) .
$$

However, if we knew that $e^{\beta x} u(x, 1), \quad e^{\beta x} u(x,-1) \in L^{2}(\mathbb{R})$ integrating forward in time the positive frequencies of $e^{\beta x} u(x, t)$ and backward in time the negative frequencies of $e^{\beta x} u(x, t)$ one gets an estimate similar to that in (1.15) with $\lambda=\beta$ and $\mathbb{G}=0$. This argument motivates the idea behind Lemma 1 and its proof.

The rest of this paper is organized as follows: Section 2 contains the results related to Hardy's uncertainty principle including a short discussion on the version of this principle in terms of the heat flow. Section 3 contains those concerned with Morgan's uncertainty principle. In section 4 we shall consider the limiting case in section 3. Also, section 4 includes the statements of some related forthcoming results. Earlier in the introduction we have discussed uniqueness results obtained under the assumption that the solution vanishes at two different times in a semispace (see [56, [33, [34, 20]). In section 2 similar uniqueness results will be established under a Gaussian decay hypothesis, in the whole space. In section 5 we shall obtain a unifying result, i.e., a uniqueness result under Gaussian decay in a semispace of $\mathbb{R}^{n}$ at two different times. The appendix contains an abstract lemma and a corollary which will be used in the previous sections.

\section{HARDY'S UNCERTAINTY PRINCIPLE}

In [28] G. H. Hardy proved the following one-dimensional $(n=1)$ result:

$$
\begin{aligned}
& \text { If } f(x)=O\left(e^{-|x|^{2} / \beta^{2}}\right), \widehat{f}(\xi)=O\left(e^{-4|\xi|^{2} / \alpha^{2}}\right) \text {, and } 1 / \alpha \beta>1 / 4, \\
& \text { then } f \equiv 0 \text {. Also, if } 1 / \alpha \beta=1 / 4, f(x) \text { is a constant multiple of } \\
& e^{-|x|^{2} / \beta^{2}} .
\end{aligned}
$$

To our knowledge the available proofs of this result and its variants use complex analysis, mainly appropriate versions of the Phragmén-Lindelöf principle. There has also been considerable interest in a better understanding of this result and on extensions of it to other settings; see [5], 6], 12, [31, and [53. In particular, the extension of Hardy's result to higher dimensions $n \geq 2$ (via Radon transform) was given in $[53$.

The formula (1.11) allows us to rewrite this uncertainty principle in terms of the solution of the IVP for the free Schrödinger equation

$$
\left\{\begin{array}{l}
\partial_{t} u=i \triangle u, \quad(x, t) \in \mathbb{R}^{n} \times(0,+\infty), \\
u(x, 0)=u_{0}(x)
\end{array}\right.
$$

in the following manner:

$$
\begin{aligned}
& \text { If } u(x, 0)=O\left(e^{-|x|^{2} / \beta^{2}}\right), u(x, T)=O\left(e^{-|x|^{2} / \alpha^{2}}\right) \text {, and } T / \alpha \beta>1 / 4, \\
& \text { then } u \equiv 0 \text {. Also, if } T / \alpha \beta=1 / 4, u \text { has as initial data } u_{0} \text { equal to }
\end{aligned}
$$$$
\text { a constant multiple of } e^{-\left(1 / \beta^{2}+i / 4 T\right)|y|^{2}} \text {. }
$$

The corresponding $L^{2}$-version of Hardy's uncertainty principle was established in [13]:

$$
\text { If } e^{|x|^{2} / \beta^{2}} f, e^{4|\xi|^{2} / \alpha^{2}} \widehat{f} \text { are in } L^{2}\left(\mathbb{R}^{n}\right) \text { and } 1 / \alpha \beta \geq 1 / 4 \text {, then } f \equiv 0 \text {. }
$$

In terms of the solution of the Schrödinger equation it states:

$$
\text { If } e^{|x|^{2} / \beta^{2}} u(x, 0), e^{|\xi|^{2} / \alpha^{2}} u(x, T) \text { are in } L^{2}\left(\mathbb{R}^{n}\right) \text { and } T / \alpha \beta \geq 1 / 4 \text {, }
$$

then $u \equiv 0$. 
More generally, it was shown in 13 that:

$$
\begin{aligned}
& \text { If } e^{|x|^{2} / \beta^{2}} f \in L^{p}\left(\mathbb{R}^{n}\right), \quad e^{4|\xi|^{2} / \alpha^{2}} \widehat{f} \in L^{q}\left(\mathbb{R}^{n}\right), p, q \in[1, \infty] \text { with at } \\
& \text { least one of them finite and } 1 / \alpha \beta \geq 1 / 4 \text {, then } f \equiv 0 \text {. }
\end{aligned}
$$

In 20] we proved a uniqueness result for solutions of (1.6) with $F$ as in (1.7) for bounded potentials $V$ verifying that either

$$
V(x, t)=V_{1}(x)+V_{2}(x, t)
$$

with $V_{1}$ real valued and

$$
\sup _{[0, T]}\left\|e^{T^{2}|x|^{2} /(\alpha t+\beta(T-t))^{2}} V_{2}(t)\right\|_{L^{\infty}\left(\mathbb{R}^{n}\right)}<+\infty,
$$

or

$$
\lim _{R \rightarrow+\infty} \int_{0}^{T}\|V(t)\|_{L^{\infty}\left(\mathbb{R}^{n} \backslash B_{R}\right)} d t=0 .
$$

More precisely, it was shown that the only solution $u \in C\left([0, T], L^{2}\left(\mathbb{R}^{n}\right)\right)$ to (1.6) with $F=V(x, t) u$, verifying

$$
\left\|e^{|x|^{2} / \beta^{2}} u(0)\right\|_{L^{2}\left(\mathbb{R}^{n}\right)}+\left\|e^{|x|^{2} / \alpha^{2}} u(T)\right\|_{L^{2}\left(\mathbb{R}^{n}\right)}<+\infty
$$

with $T / \alpha \beta>1 / 2$ and $V$ satisfying one of the above conditions, is the zero solution. Notice that this result differs by a factor of $1 / 2$ from that for the solution of the free Schrödinger equation given by the $L^{2}$-version of the Hardy uncertainty principle described above $(T / \alpha \beta \geq 1 / 4)$.

In 22 we showed that the optimal version of Hardy's uncertainty principle in terms of $L^{2}$-norms, as established in [13], holds for solutions of

$$
\partial_{t} u=i(\triangle u+V(x, t) u), \quad(x, t) \in \mathbb{R}^{n} \times[0, T],
$$

such that (2.2) holds with $T / \alpha \beta>1 / 4$ and for many general bounded potentials $V(x, t)$, while it fails for some complex-valued potentials in the endpoint case, $T / \alpha \beta=1 / 4$.

Theorem 3. Let $\left.u \in C([0, T]): L^{2}\left(\mathbb{R}^{n}\right)\right)$ be a solution of equation (2.3). If there exist positive constants $\alpha$ and $\beta$ such that $T / \alpha \beta>1 / 4$, and

$$
\left\|e^{|x|^{2} / \beta^{2}} u(0)\right\|_{L^{2}\left(\mathbb{R}^{n}\right)}, \quad\left\|e^{|x|^{2} / \alpha^{2}} u(T)\right\|_{L^{2}\left(\mathbb{R}^{n}\right)}<\infty,
$$

and the potential $V$ is bounded, and either $V(x, t)=V_{1}(x)+V_{2}(x, t)$ with $V_{1}$ real valued and

$$
\sup _{[0, T]}\left\|e^{T^{2}|x|^{2} /(\alpha t+\beta(T-t))^{2}} V_{2}(t)\right\|_{L^{\infty}\left(\mathbb{R}^{n}\right)}<+\infty
$$

or

$$
\lim _{R \rightarrow+\infty}\|V\|_{L^{1}\left([0, T], L^{\infty}\left(\mathbb{R}^{n} \backslash B_{R}\right)\right.}=0,
$$

then $u \equiv 0$.

We remark that there are no assumptions on the size of the potential in the given class or on the dimension, and that we do not assume any decay of the gradient, neither of the solutions nor of the time-independent potential or any a priori regularity on this potential or the solution. 
Theorem 4. Assume that $T / \alpha \beta=1 / 4$. Then there is a smooth complex-valued potential $V$ verifying

$$
|V(x, t)| \lesssim \frac{1}{1+|x|^{2}},(x, t) \in \mathbb{R}^{n} \times[0, T],
$$

and a nonzero smooth function $u \in C^{\infty}\left([0, T], \mathcal{S}\left(\mathbb{R}^{n}\right)\right)$ solution of (2.3) such that

$$
\left\|e^{|x|^{2} / \beta^{2}} u(0)\right\|_{L^{2}\left(\mathbb{R}^{n}\right)}, \quad\left\|e^{|x|^{2} / \alpha^{2}} u(T)\right\|_{L^{2}\left(\mathbb{R}^{n}\right)}<\infty .
$$

Our proof of Theorem 3 does not use any complex analysis, giving, in particular, a new proof (up to the endpoint) of the $L^{2}$-version of Hardy's uncertainty principle for the Fourier transform. It is based on Carleman estimates for certain evolutions. More precisely, it is based on the convexity and log-convexity properties present for the solutions of these evolutions. Thus, the convexity and log-convexity of appropriate $L^{2}$-quantities play the role of the Phragmén-Lindelöf principle. We observe that the product of log-convex functions is log-convex, which, roughly speaking, replaces the fact that the product of analytic functions is analytic.

In 11] in collaboration with M. Cowling, we gave new proofs, based only on real variable techniques, of both the $L^{2}$-version of the Hardy uncertainty principle and the original Hardy's uncertainty principle $\left(L^{\infty}\right) n$-dimensional version for the Fourier transform as stated at the beginning of this section, including the endpoint case $1 / \alpha \beta=1 / 4$.

Returning to Theorem 3 as a by-product of our proof, we obtain the following optimal interior estimate for the Gaussian decay of solutions to (2.3).

Theorem 5. Assume that $u$ and $V$ verify the hypothesis in Theorem 3 and $T / \alpha \beta \leq 1 / 4$. Then,

$$
\begin{aligned}
& \sup _{[0, T]}\left\|e^{a(t)|x|^{2}} u(t)\right\|_{L^{2}\left(\mathbb{R}^{n}\right)}+\left\|\sqrt{t(T-t)} \nabla\left(e^{\left(a(t)+\frac{i \dot{a}(t)}{8 a(t)}\right)|x|^{2}} u\right)\right\|_{L^{2}\left(\mathbb{R}^{n} \times[0, T]\right)} \\
& \leq N\left[\left\|e^{|x|^{2} / \beta^{2}} u(0)\right\|_{L^{2}\left(\mathbb{R}^{n}\right)}+\left\|e^{|x|^{2} / \alpha^{2}} u(T)\right\|_{L^{2}\left(\mathbb{R}^{n}\right)}\right]
\end{aligned}
$$

where

$$
a(t)=\frac{\alpha \beta R T}{2(\alpha t+\beta(T-t))^{2}+2 R^{2}(\alpha t-\beta(T-t))^{2}},
$$

$R$ is the smallest root of the equation

$$
\frac{T}{\alpha \beta}=\frac{R}{2\left(1+R^{2}\right)}
$$

and $N$ depends on $T, \alpha, \beta$ and the conditions on the potential $V$ in Theorem 3 ,

One has that $1 / a(t)$ is convex and attains its minimum value in the interior of $[0, T]$, when

$$
|\alpha-\beta|<R^{2}(\alpha+\beta) .
$$

To see the optimality of Theorem 5, we write

$$
u_{R}(x, t)=R^{-\frac{n}{2}}\left(t-\frac{i}{R}\right)^{-\frac{n}{2}} e^{-\frac{|x|^{2}}{4 i\left(t-\frac{i}{R}\right)}}=(R t-i)^{-\frac{n}{2}} e^{-\frac{\left(R-i R^{2} t\right)}{4\left(1+R^{2} t^{2}\right)}|x|^{2}},
$$

which is a free wave (i.e., $V \equiv 0$, in (2.3) ) satisfying in $\mathbb{R}^{n} \times[-1,1]$ the corresponding time translated conditions in Theorem 5 with $T=2$ and

$$
\frac{1}{\beta^{2}}=\frac{1}{\alpha^{2}}=\mu=\frac{R}{4\left(1+R^{2}\right)} \leq \frac{1}{8} \text {. }
$$


Moreover,

$$
\frac{R}{4\left(1+R^{2} t^{2}\right)}
$$

is increasing in the $R$-variable, when $0<R \leq 1$ and $-1 \leq t \leq 1$.

Our improvement over the results in [16] and [20] is a consequence of the possibility of extending the following argument (for the case of free waves) to prove Theorem 3 (a nonfree wave case):

We recall the conformal or Appell transformation: If $u(y, s)$ verifies

$$
\partial_{s} u=i(\triangle u+V(y, s) u+F(y, s)), \quad(y, s) \in \mathbb{R}^{n} \times[0,1],
$$

and $\alpha$ and $\beta$ are positive, then

$$
\widetilde{u}(x, t)=\left(\frac{\sqrt{\alpha \beta}}{\alpha(1-t)+\beta t}\right)^{\frac{n}{2}} u\left(\frac{\sqrt{\alpha \beta} x}{\alpha(1-t)+\beta t}, \frac{\beta t}{\alpha(1-t)+\beta t}\right) e^{\frac{(\alpha-\beta)|x|^{2}}{4 i(\alpha(1-t)+\beta t)}}
$$

verifies

$$
\partial_{t} \widetilde{u}=i(\triangle \widetilde{u}+\widetilde{V}(x, t) \widetilde{u}+\widetilde{F}(x, t)), \text { in } \mathbb{R}^{n} \times[0,1],
$$

with

$$
\widetilde{V}(x, t)=\frac{\alpha \beta}{(\alpha(1-t)+\beta t)^{2}} V\left(\frac{\sqrt{\alpha \beta} x}{\alpha(1-t)+\beta t}, \frac{\beta t}{\alpha(1-t)+\beta t}\right)
$$

and

$$
\widetilde{F}(x, t)=\left(\frac{\sqrt{\alpha \beta}}{\alpha(1-t)+\beta t}\right)^{\frac{n}{2}+2} F\left(\frac{\sqrt{\alpha \beta} x}{\alpha(1-t)+\beta t}, \frac{\beta t}{\alpha(1-t)+\beta t}\right) e^{\frac{(\alpha-\beta)|x|^{2}}{4 i(\alpha(1-t)+\beta t)}} .
$$

Thus, to prove Theorem 3 for free waves, it suffices to consider $u \in C\left([-1,1], L^{2}\left(R^{n}\right)\right)$ being a solution of

$$
\partial_{t} u-=i \triangle u, \quad(x, t) \in R \times[-1,1]
$$

and

$$
\left\|e^{\mu|x|^{2}} u(-1)\right\|_{L^{2}\left(R^{n}\right)}+\left\|e^{\mu|x|^{2}} u(1)\right\|_{L^{2}\left(\mathbb{R}^{n}\right)}<+\infty,
$$

for some $\mu>0$.

The main idea consists of showing that either $u \equiv 0$ or there is a function $\theta_{R}:[-1,1] \longrightarrow[0,1]$ such that

$$
\left\|e^{\frac{R|x|^{2}}{4\left(1+R^{2} t^{2}\right)}} u(t)\right\|_{L^{2}\left(R^{n}\right)} \leq\left\|e^{\mu|x|^{2}} u(-1)\right\|_{L^{2}\left(R^{n}\right)}^{\theta_{R}(t)}\left\|e^{\mu|x|^{2}} u(1)\right\|_{L^{2}\left(\mathbb{R}^{n}\right)}^{1-\theta_{R}(t)},
$$

where $R$ is the smallest root of the equation

$$
\mu=\frac{R}{4\left(1+R^{2}\right)} .
$$

This gives the optimal improvement of the Gaussian decay of a free wave verifying (2.13), and we also see that if $\mu>1 / 8$, then $u$ is zero.

The proof of these facts relies on new logarithmic convexity properties of free waves verifying (2.13) and on those already established in [20. In [20, Theorem 3], the positivity of the space-time commutator of the symmetric and skew-symmetric parts of the operator,

$$
e^{\mu|x|^{2}}\left(\partial_{t}-i \triangle\right) e^{-\mu|x|^{2}}
$$

is used to prove that $\left\|e^{\mu|x|^{2}} u(t)\right\|_{L^{2}\left(\mathbb{R}^{n}\right)}$ is logarithmically convex in $[-1,1]$. More precisely, defining

$$
f(x, t)=e^{\mu|x|^{2}} u(x, t)=e^{i t \Delta} u_{0}(x),
$$


it follows that

$$
e^{\mu|x|^{2}}\left(\partial_{t}-i \triangle\right) u=e^{\mu|x|^{2}}\left(\partial_{t}-i \triangle\right)\left(e^{-\mu|x|^{2}} f\right)=\partial_{t} f-\mathcal{S} f-\mathcal{A} f,
$$

where $\mathcal{S}$ is symmetric and $\mathcal{A}$ skew-symmetric with

$$
\mathcal{S}=-i \mu(4 x \cdot \nabla+2 n), \quad \mathcal{A}=i\left(\Delta+4 \mu^{2}|x|^{2}\right),
$$

so that

$$
[\mathcal{S} ; \mathcal{A}]=-8 \mu(\nabla \cdot I \nabla)+16 \mu^{2}|x|^{2} .
$$

Formally, using the abstract Lemma 3 (see the appendix) and the Heisenberg inequality

$$
\|f\|_{L^{2}\left(\mathbb{R}^{n}\right)}^{2} \leq \frac{2}{n}\||x| f\|_{L^{2}\left(\mathbb{R}^{n}\right)}\|\nabla f\|_{L^{2}\left(\mathbb{R}^{n}\right)}
$$

whose proof follows by integration by parts, one sees that

$$
H(t)=\|f(t)\|_{L^{2}\left(\mathbb{R}^{n}\right)}^{2}=\left\|e^{\mu|x|^{2}} u(t)\right\|_{L^{2}\left(\mathbb{R}^{n}\right)}
$$

is logarithmically convex so

$$
\left\|e^{\mu|x|^{2}} u(t)\right\|_{L^{2}\left(\mathbb{R}^{n}\right)} \leq\left\|e^{\mu|x|^{2}} u(-1)\right\|_{L^{2}\left(R^{n}\right)}^{\frac{1-t}{2}}\left\|e^{\mu|x|^{2}} u(1)\right\|_{L^{2}\left(\mathbb{R}^{n}\right)}^{\frac{1+t}{2}},
$$

when $-1 \leq t \leq 1$.

Setting $a_{1} \equiv \mu$, we begin an iterative process, where at the $k$ th step, we have $k$ smooth even functions, $a_{j}:[-1,1] \longrightarrow(0,+\infty), 1 \leq j \leq k$, such that

$$
\begin{gathered}
\mu \equiv a_{1}<a_{2}<\cdots<a_{k} \in(-1,1), \\
F\left(a_{i}\right)>0, a_{j}(1)=\mu, j=1, \ldots, k,
\end{gathered}
$$

where

$$
F(a)=\frac{1}{a}\left(\ddot{a}-\frac{3 \dot{a}^{2}}{2 a}+32 a^{3}\right)
$$

and functions $\theta_{j}:[-1,1] \longrightarrow[0,1], 1 \leq j \leq k$, such that for $t \in[.1,1]$

$$
\left\|e^{a_{j}(t)|x|^{2}} u(t)\right\|_{L^{2}\left(R^{n}\right)} \leq\left\|e^{\mu|x|^{2}} u(-1)\right\|_{L^{2}\left(R^{n}\right)}^{\theta_{j}(t)}\left\|e^{\mu|x|^{2}} u(1)\right\|_{L^{2}\left(\mathbb{R}^{n}\right)}^{1-\theta_{j}(t)} .
$$

These estimates follow from the construction of the functions $a_{i}$, while the method strongly relies on the following formal convexity properties of free waves:

$$
\begin{gathered}
\partial_{t}\left(\frac{1}{a} \partial_{t} \log H_{b}\right) \geq-\frac{2 \ddot{b}^{2}|\xi|^{2}}{F(a)}, \\
\partial_{t}\left(\frac{1}{a} \partial_{t} H\right) \geq \epsilon_{a} \int_{R^{n}} e^{a|x|^{2}}\left(|\nabla u|^{2}+|x|^{2}|u|^{2}\right) d x,
\end{gathered}
$$

where

$$
H_{b}(t)=\left\|e^{a(t)|x+b(t) \xi|^{2}} u(t)\right\|_{L^{2}\left(R^{n}\right)}^{2}, H(t)=\left\|e^{a(t)|x|^{2}} u(t)\right\|_{L^{2}\left(\mathbb{R}^{n}\right)}^{2},
$$

$\xi \in \mathbb{R}^{n}$ and $a, b:[-1,1] \longrightarrow R$ are smooth functions with

$$
a>0, \quad F(a)>0 \quad \text { in }[-1,1] .
$$

Once the $k$ th step is completed, we take $a=a_{k}$ in (2.16) with a certain choice of $b=b_{k}$, verifying $b(-1)=b(1)=0$, and then a certain test is performed. When the answer to the test is positive, it follows that $u \equiv 0$. Otherwise, the logarithmic convexity associated to (2.16) allows us to find a new smooth function $a_{k+1}$ in $[-1,1]$ with

$$
a_{1}<a_{2}<\cdots<a_{k}<a_{k+1},(-1,1),
$$

and verifying the same properties as $a_{1}, \ldots, a_{k}$. 
When the process is infinite, we have (2.15) for all $k \geq 1$ and there are two possibilities:

$$
\text { either } \quad \lim _{k \rightarrow+\infty} a_{k}(0)=+\infty \quad \text { or } \quad \lim _{k \rightarrow+\infty} a_{k}(0)<+\infty .
$$

In the first case and (2.15) one has that $u \equiv 0$, while in the second, the sequence $a_{k}$ is shown to converge to an even function $a$ verifying

$$
\left\{\begin{array}{l}
\ddot{a}-\frac{3 \dot{a}^{2}}{2 a}+32 a^{3}=0, \quad[-1,1], \\
a(1)=\mu .
\end{array}\right.
$$

Because

$$
a(t)=\frac{R}{4\left(1+R^{2} t^{2}\right)}, \quad R \in \mathbb{R}^{+},
$$

are all the possible even solutions of this equation, $a$ must be one of them, and

$$
\mu=\frac{R}{4\left(1+R^{2}\right)}
$$

for some $R>0$. In particular, $u \equiv 0$, when $\mu>1 / 8$.

As was already mentioned above, our proof of Theorem 3 (the case of nonzero potentials $V=V(x, t))$ is based on the extension of the above convexity properties to the nonfree case.

Theorem 4 establishes the sharpness of the result in Theorem 3 by giving an example of a complex valued potential $V(x, t)$ verifying (2.1) and a nontrivial solution $u \in C\left([0, T]: L^{2}\left(\mathbb{R}^{n}\right)\right)$ of (2.3) for which (2.2) holds with $T / \alpha \beta=1 / 4$. Thus, one may ask, Is it possible to construct a real-valued potential $V(x, t)$ verifying the same properties, i.e., satisfying (2.1) and having a nontrivial solution $u \in C\left([0, T]: L^{2}\left(\mathbb{R}^{n}\right)\right)$ of (2.3) such that (2.2) holds with $T / \alpha \beta=1 / 4$ ?

The same question concerning the sharpness of the above result presents itself in the case of time-independent potentials $V=V(x)$. In this regard, we consider the stationary problem

$$
\Delta w+V(x) w=0, \quad x \in \mathbb{R}^{n}, V \in L^{\infty}\left(\mathbb{R}^{n}\right),
$$

and recall V. Z. Meshkov's result in [45]:

If $w \in H_{\mathrm{loc}}^{2}\left(\mathbb{R}^{n}\right)$ is a solution of (2.19) such that

$$
\int_{\mathbb{R}^{n}} e^{a|x|^{4 / 3}}|w(x)|^{2} d x<\infty, \quad \forall a>0
$$

then $u \equiv 0$.

Moreover, it was also proved in [45] that for complex potentials $V$, the exponent $4 / 3$ in (2.20) is optimal. However, it has been conjectured that for real-valued potentials the optimal exponent should be 1 (see also 7] for a quantitative form of these results and applications to Anderson localization of Bernoulli models).

More generally, it was established in [23] (see also [14]):

If $w \in H_{\mathrm{loc}}^{2}\left(\mathbb{R}^{n}\right)$ is a solution of (2.19) with a complex-valued potential $V$ satisfying

$$
V(x)=V_{1}(x)+V_{2}(x)
$$

such that

$$
\left|V_{1}(x)\right| \leq \frac{c_{1}}{\left(1+|x|^{2}\right)^{\alpha / 2}}, \quad \alpha \in[0,1 / 2)
$$


and $V_{2}$ is real valued supported in $\{x:|x| \geq 1\}$ such that

$$
-\left(\partial_{r} V_{2}(x)\right)^{-}<\frac{c_{2}}{|x|^{2 \alpha}}, \quad a^{-}=\min \{a ; 0\},
$$

then there exists $a=a\left(\|V\|_{\infty} ; c_{1} ; c_{2} ; \alpha\right)>0$ such that if

$$
\int_{\mathbb{R}^{n}} e^{a|x|^{r}}|w(x)|^{2} d x<\infty, \quad r=(4-2 \alpha) / 3,
$$

then $u \equiv 0$.

In addition, one can take the value $r=1$ in $(2.20)$ by assuming $\alpha>1 / 2$ in (2.21).

It was also proved in [14] that for complex potentials, these results for $\alpha \in[0,1 / 2)$ are sharp.

By noticing that given a solution $\phi(x)$ of the eigenvalue problem

$$
\Delta \phi+\widetilde{V}(x) \phi=\lambda \phi, \quad x \in \mathbb{R}^{n},
$$

with $\lambda \in \mathbb{R}$, if $V(x)=\widetilde{V}(x)+\lambda$ satisfies the hypothesis of the previous result since

$$
u(x, t)=e^{i t \lambda} \phi(x),
$$

solves the evolution equation

$$
\partial_{t} u=i(\Delta u+\tilde{V}(x) u), \quad x \in \mathbb{R}^{n}, t \in \mathbb{R},
$$

then one gets a lower bound for the value of the strongest possible decay rate of nontrivial solutions $u(x, t)$ of (2.24) at two different times.

As a direct consequence of Theorem 3 we have the following application concerning the uniqueness of solutions for semilinear equations of the form (1.6) with $F$ as in (1.8).

Theorem 6. Let $u_{1}$ and $u_{2}$ be strong solutions in $C\left([0, T], H^{k}\left(\mathbb{R}^{n}\right)\right), k>n / 2$ of the equation (1.6) with $F$ as in (1.8) such that $F \in C^{k}$ and $F(0)=\partial_{u} F(0)=$ $\partial_{\bar{u}} F(0)=0$. If there are $\alpha$ and $\beta$ positive with $T / \alpha \beta>1 / 4$ such that

$$
e^{|x|^{2} / \beta^{2}}\left(u_{1}(0)-u_{2}(0)\right), e^{|x|^{2} / \alpha^{2}}\left(u_{1}(T)-u_{2}(T)\right) \in L^{2}\left(\mathbb{R}^{n}\right),
$$

then $u_{1} \equiv u_{2}$.

In Theorem 6 we did not attempt to optimize the regularity assumption on the solutions $u_{1}, u_{2}$.

By fixing $u_{2} \equiv 0$ Theorem 6 provides a restriction on the possible decay at two different times of a nontrivial solution $u_{1}$ of equation (1.6) with $F$ as in (1.8). It is an open question to determine the optimality of this kind of result. More precisely, for the standard semilinear Schrödinger equations

$$
\partial_{t} u=i\left(\Delta u+|u|^{\gamma-1} u\right), \quad \gamma>1,
$$

one has the standing wave solutions

$$
u(x, t)=e^{\omega t} \varphi(x), \omega>0,
$$

where $\varphi$ is the unique (up to translation) positive solution of the elliptic problem

$$
-\Delta \varphi+\omega \varphi=|\varphi|^{\gamma-1} \varphi
$$

which has a linear exponential decay, i.e.,

$$
\varphi(x)=O\left(e^{-c|x|}\right), \text { as }|x| \rightarrow \infty,
$$


for an appropriate value of $c>0$ (see [54, 3], 4], and 42]). Whether or not these standing waves are the solutions of (2.25) having the strongest possible decay at two different times is an open question.

Hardy's uncertainty principle also admits a formulation in terms of the heat equation

$$
\partial_{t} u=\Delta u, \quad t>0, \quad x \in \mathbb{R}^{n},
$$

whose solution with data $u(x, 0)=u_{0}(x)$ can be written as

$$
u(x, t)=e^{t \Delta} u_{0}(x)=\int_{\mathbb{R}^{n}} \frac{e^{-|x-y|^{2} / 4 t}}{(4 \pi)^{n / 2}} u_{0}(y) d y .
$$

More precisely, Hardy's uncertainty principle can restated in the following equivalent forms:

(i) If $u_{0} \in L^{2}\left(\mathbb{R}^{n}\right)$ and there exists $T>0$ such that $e^{|x|^{2} /\left(\delta^{2} T\right)} e^{T \Delta} u_{0} \in$ $L^{2}\left(\mathbb{R}^{n}\right)$ for some $\delta \leq 2$, then $u_{0} \equiv 0$.

(ii) If $u_{0} \in \mathcal{S}\left(\mathbb{R}^{n}\right)$ (tempered distribution) and there exists $T>0$ such that $e^{|x|^{2} /\left(\delta^{2} T\right)} e^{T \Delta} u_{0} \in L^{\infty}\left(\mathbb{R}^{n}\right)$ for some $\delta<2$, then $u_{0} \equiv 0$. Moreover, if $\delta=2$, then $u_{0}$ is a constant multiple of the Dirac delta measure.

In fact, applying Hardy's uncertainty principle to $e^{T \triangle} u_{0}$, one has that $e^{\frac{|x|^{2}}{\delta^{2} T}} e^{T \triangle} u_{0}$ and $e^{T|\xi|^{2}} \widehat{e^{T \triangle} u_{0}}=\widehat{u}_{0}$ in $L^{2}\left(\mathbb{R}^{n}\right)$ with $2 \delta \leq 4$ implies $e^{\triangle} u_{0} \equiv 0$. Then, backward uniqueness arguments (see for example [44, Chapter 3, Theorem 11]) show that $u_{0} \equiv 0$.

In 20] we proved the following weaker extension of this result for parabolic operators with lower order variable coefficients:

Theorem 7. Let $u \in C\left([0,1]: L^{2}\left(\mathbb{R}^{n}\right)\right) \cap L^{2}\left([0, T]: H^{1}\left(\mathbb{R}^{n}\right)\right)$ be a solution of the IVP

where

$$
\left\{\begin{array}{l}
\partial_{t} u=\Delta u+V(x, t) u, \text { in } \mathbb{R}^{n} \times(0,1], \\
u(x, 0)=u_{0}(x)
\end{array}\right.
$$

$$
V \in L^{\infty}\left(\mathbb{R}^{n} \times[0,1]\right)
$$

If

for some $\delta<1$, then $u_{0} \equiv 0$.

$$
u_{0} \quad \text { and } \quad e^{\frac{|x|^{2}}{\delta^{2}}} u(1) \in L^{2}\left(\mathbb{R}^{n}\right)
$$

It is natural to expect that Hardy's uncertainty principle holds in this context with bounded potentials $V$ and with the parameter $\delta$ verifying the condition of the free case, i.e., $\delta \leq 2$.

Earlier results in these directions, addressing a question of Landis and Oleinik [43, were obtained in [18] and [49].

\section{UNCERTAINTY PRINCIPLE OF MORGAN TYPE}

In 46] G. W. Morgan proved the following uncertainty principle:

If $f(x)=O\left(e^{-\frac{a^{p}|x|^{p}}{p}}\right), 1<p \leq 2$ and $\widehat{f}(\xi)=O\left(e^{-\frac{(b+\epsilon)^{q}|\xi|^{q}}{q}}\right), 1 / p+$ $1 / q=1, \epsilon>0$, with

$$
a b>\left|\cos \left(\frac{p \pi}{2}\right)\right|
$$

then $f \equiv 0$. 
In 31] Beurling and Hörmander showed:

If $f \in L^{1}(\mathbb{R})$ and

$$
\int_{\mathbb{R}} \int_{\mathbb{R}}|f(x)||\widehat{f}(\xi)| e^{|x \xi|} d x d \xi<\infty,
$$

then $f \equiv 0$.

This result was extended to higher dimensions $n \geq 2$ in [6] and [48]:

If $f \in L^{2}\left(\mathbb{R}^{n}\right), n \geq 2$ and

$$
\int_{\mathbb{R}^{n}} \int_{\mathbb{R}^{n}}|f(x)||\widehat{f}(\xi)| e^{|x \cdot \xi|} d x d \xi<\infty,
$$

then $\equiv 0$.

We observe that from (3.1) and (3.2) it follows that:

$$
\text { If } p \in(1,2], 1 / p+1 / q=1, a, b>0 \text {, and }
$$

$$
\int_{\mathbb{R}^{n}}|f(x)| e^{\frac{a^{p}|x|^{p}}{p}} d x+\int_{\mathbb{R}^{n}}|\widehat{f}(\xi)| e^{\frac{b^{q}|\xi|^{q}}{q}} d \xi<\infty,
$$

then $a b \geq 1 \Rightarrow f \equiv 0$.

Notice that in the case $p=q=2$ this gives us an $L^{1}$-version of Hardy's uncertainty result discussed above, and for $p<2$ an $n$-dimensional $L^{1}$-version of Morgan's uncertainty principle.

In the one-dimensional case $(n=1)$, the optimal $L^{1}$-version of Morgan's result in (3.3),

$$
\int_{\mathbb{R}}|f(x)| e^{\frac{a^{p}|x|^{p}}{p}} d x+\int_{\mathbb{R}}|\widehat{f}(\xi)| e^{\frac{b^{q}|\xi|^{q}}{q}} d \xi<\infty, \quad a b>\left|\cos \left(\frac{p \pi}{2}\right)\right| \Rightarrow f \equiv 0 .
$$

was established in [6] and 2] (for further results, see [5] and references therein). A sharp condition for $a, b, p$ in (3.4) in higher dimension seems to be unknown. However, in [6] it was shown:

If $f \in L^{2}\left(\mathbb{R}^{n}\right), 1<p \leq 2$ and $1 / p+1 / q=1$ are such that for some $j=1, \ldots, n$,

$$
\int_{\mathbb{R}^{n}}|f(x)| e^{\frac{a^{p}\left|x_{j}\right|^{p}}{p}} d x<\infty+\int_{\mathbb{R}^{n}}|\widehat{f}(\xi)| e^{\frac{b^{q}\left|\xi_{j}\right|^{q}}{q}} d \xi<\infty .
$$

If $a b>\left|\cos \left(\frac{p \pi}{2}\right)\right|$, then $f \equiv 0$.

If $a b<\left|\cos \left(\frac{p \pi}{2}\right)\right|$, then there exist nontrivial functions satisfying (3.5).

Using (1.11), the above result can be stated in terms of the solution of the free Schrödinger equation. In particular, (3.3) can be rewritten as:

$$
\text { If } u_{0} \in L^{1}(\mathbb{R}) \text { or } u_{0} \in L^{2}\left(\mathbb{R}^{n}\right) \text {, if } n \geq 2 \text {, and for some } t \neq 0
$$

$$
\int_{\mathbb{R}^{n}}\left|u_{0}(x)\right| e^{\frac{a^{p}|x|^{p}}{p}} d x+\int_{\mathbb{R}^{n}}\left|e^{i t \Delta} u_{0}(x)\right| e^{\frac{b^{q}|x|^{q}}{q(2 t)^{q}}} d x<\infty,
$$

with

$$
a b>\left|\cos \left(\frac{p \pi}{2}\right)\right| \quad \text { if } n=1, \quad \text { and } \quad a b>1 \quad \text { if } \quad n \geq 2,
$$

then $u_{0} \equiv 0$. 
Related with Morgan's uncertainty principle, one has the following result due to Gel'fand and Shilov. In [26] they considered the class $Z_{p}^{p}, p>1$, defined as the space of all functions $\varphi\left(z_{1}, \ldots, z_{n}\right)$ which are analytic for all values of $z_{1}, \ldots, z_{n} \in \mathbb{C}$ and such that

$$
\left|\varphi\left(z_{1}, \ldots, z_{n}\right)\right| \leq C_{0} e^{\sum_{j=1}^{n} \epsilon_{j} C_{j}\left|z_{j}\right|^{p}},
$$

where the $C_{j}, j=0,1, \ldots, n$ are positive constants and $\epsilon_{j}=1$ for $z_{j}$ nonreal and $\epsilon_{j}=-1$ for $z_{j}$ real, $j=1, \ldots, n$, and showed that the Fourier transform of the function space $Z_{p}^{p}$ is the space $Z_{q}^{q}$, with $1 / p+1 / q=1$.

Notice that the class $Z_{p}^{p}$ with $p \geq 2$ is closed with respect to multiplication by $e^{i c|x|^{2}}$. Thus, if $u_{0} \in Z_{p}^{p}, p \geq 2$, then by (1.11) one has that

$$
\left|e^{i t \Delta} u_{0}(x)\right| \leq d(t) e^{-a(t)|x|^{q}},
$$

for some functions $d, a: \mathbb{R} \rightarrow(0, \infty)$.

In 21 the following results were established:

Theorem 8. Given $p \in(1,2)$ there exists $M_{p}>0$ such that for any solution $u \in C\left([0,1]: L^{2}\left(\mathbb{R}^{n}\right)\right)$ of

$$
\partial_{t} u=i(\triangle u+V(x, t) u), \quad \text { in } \quad \mathbb{R}^{n} \times[0,1],
$$

with $V=V(x, t)$ complex valued, bounded (i.e., $\|V\|_{L^{\infty}\left(\mathbb{R}^{n} \times[0,1]\right)} \leq C$ ) and

$$
\lim _{R \rightarrow+\infty}\|V\|_{L^{1}\left([0,1]: L^{\infty}\left(\mathbb{R}^{n} \backslash B_{R}\right)\right)}=0,
$$

satisfying that for some constants $a_{0}, a_{1}, a_{2}>0$

$$
\int_{\mathbb{R}^{n}}|u(x, 0)|^{2} e^{2 a_{0}|x|^{p}} d x<\infty
$$

and for any $k \in \mathbb{Z}^{+}$

$$
\int_{\mathbb{R}^{n}}|u(x, 1)|^{2} e^{2 k|x|^{p}} d x<a_{2} e^{2 a_{1} k^{q /(q-p)}}
$$

$1 / p+1 / q=1$, if

$$
a_{0} a_{1}^{(p-2)}>M_{p}
$$

then $u \equiv 0$.

Corollary 1. Given $p \in(1,2)$ there exists $N_{p}>0$ such that if $u \in C\left([0,1]: L^{2}\left(\mathbb{R}^{n}\right)\right)$ is a solution of

$$
\partial_{t} u=i(\Delta u+V(x, t) u)
$$

with $V=V(x, t)$ complex valued, bounded (i.e., $\|V\|_{L^{\infty}\left(\mathbb{R}^{n} \times[0,1]\right)} \leq C$ ) and

$$
\lim _{R \rightarrow \infty} \int_{0}^{1} \sup _{|x|>R}|V(x, t)| d t=0,
$$

and there exist $\alpha, \beta>0$ such that

$$
\int_{\mathbb{R}^{n}}|u(x, 0)|^{2} e^{2 \alpha^{p}|x|^{p} / p} d x+\int_{\mathbb{R}^{n}}|u(x, 1)|^{2} e^{2 \beta^{q}|x|^{q} / q} d x<\infty,
$$

$1 / p+1 / q=1$, with

$$
\alpha \beta>N_{p},
$$

then $u \equiv 0$. 
As a consequence of Corollary 1 one obtains the following result concerning the uniqueness of solutions for the semilinear equations (1.6) with $F$ as in (1.8)

$$
i \partial_{t} u+\triangle u=F(u, \bar{u}) .
$$

Theorem 9. Given $p \in(1,2)$ there exists $N_{p}>0$ such that if

$$
u_{1}, u_{2} \in C\left([0,1]: H^{k}\left(\mathbb{R}^{n}\right)\right)
$$

are strong solutions of (3.13) with $k \in \mathbb{Z}^{+}, k>n / 2, F: \mathbb{C}^{2} \rightarrow \mathbb{C}, F \in C^{k}$, and $F(0)=\partial_{u} F(0)=\partial_{\bar{u}} F(0)=0$, and there exist $\alpha, \beta>0$ such that

$$
e^{\alpha^{p}|x|^{p} / p}\left(u_{1}(0)-u_{2}(0)\right), \quad e^{\beta^{q}|x|^{q} / q}\left(u_{1}(1)-u_{2}(1)\right) \in L^{2}\left(\mathbb{R}^{n}\right),
$$

$1 / p+1 / q=1$, with

$$
\alpha \beta>N_{p},
$$

then $u_{1} \equiv u_{2}$.

Notice that the conditions (3.10) and (3.12) are independent of the size of the potential and there is not any a priori regularity assumption on the potential $V(x, t)$.

The result in 6] (see (3.5)) can be extended to our setting with a nonoptimal constant. More precisely,

Corollary 2. The conclusions in Corollary 1 still hold with a different constant $N_{p}>0$ if one replaces the hypothesis (3.11) by the following one-dimensional version

$$
\int_{\mathbb{R}^{n}}|u(x, 0)|^{2} e^{2 \alpha^{p}\left|x_{j}\right|^{p} / p} d x<\infty+\int_{\mathbb{R}^{n}}|u(x, 1)|^{2} e^{2 \beta^{q}\left|x_{j}\right|^{q} / q} d x<\infty,
$$

for some $j=1, \ldots, n$.

Similarly, the nonlinear version of Theorem 9 still holds, with different constant $N_{p}>0$, if one replaces the hypothesis (3.14) by

$$
e^{\alpha^{p}\left|x_{j}\right|^{p} / p}\left(u_{1}(0)-u_{2}(0)\right), \quad e^{\beta^{q}\left|x_{j}\right|^{q} / q}\left(u_{1}(1)-u_{2}(1)\right) \in L^{2}\left(\mathbb{R}^{n}\right),
$$

for $j=1, \ldots, n$.

In [21] we did not attempt to give an estimate of the universal constant $N_{p}$.

The limiting case $p=1$ will be considered in the next section.

The main idea in the proof of these results is to combine an upper estimate with a lower one to obtain the desired result. The upper estimate is based on the decay hypothesis on the solution at two different times (see Lemma 1). In previous works we had been able to establish these estimates from assumptions that at time $t=0$ and $t=1$ involving the same weight. However, in our case (Corollary 1) we have different weights at time $t=0$ and $t=1$. To overcome this difficulty, we carry out the details with the weight $e^{a_{j}|x|^{p}}, 1<p<2, j=0$ at $t=0$ and $j=1$ at $t=1$, with $a_{0}$ fixed and $a_{1}=k \in \mathbb{Z}^{+}$as in (3.9). Although the powers $|x|^{p}$ in the exponential are equal at time $t=0$ and $t=1$ to apply our estimate (Lemma 1) we also need to have the same constant in front of them. To achieve this we apply the conformal or Appell transformation described above, to get solutions and potentials, whose bounds depend on $k \in \mathbb{Z}^{+}$. Thus we have to consider a family of solutions and obtain estimates on their asymptotic value as $k \uparrow \infty$.

The proof of the lower estimate is based on the positivity of the commutator operator obtained by conjugating the equation with the appropriate exponential weight (see Lemma 3 in the appendix). 


\section{Paley-Wiener theorem and uncertainty Principle OF INGHAM TYPE}

This section is concerned with the limiting case $p=1$ in the previous section.

It is easy to see that if $f \in L^{1}\left(\mathbb{R}^{n}\right)$ is nonzero and has compact support, then $\widehat{f}$ cannot satisfy a condition of the type $\widehat{f}(y)=O\left(e^{-\epsilon|y|}\right)$ for any $\epsilon>0$. However, it may be possible to have $f \in L^{1}\left(\mathbb{R}^{n}\right)$ a nonzero function with compact support, such that $\widehat{f}(\xi)=O\left(e^{-\epsilon(y)|y|}\right), \epsilon(y)$ being a positive function tending to zero as $|y| \rightarrow \infty$.

In the one-dimensional case $(n=1)$ soon after Hardy's result described above, A. E. Ingham 32 proved the following:

There exists $f \in L^{1}(\mathbb{R})$ nonzero, even, vanishing outside an interval such that $\widehat{f}(y)=O\left(e^{-\epsilon(y)|y|}\right)$ with $\epsilon(y)$ being a positive function tending to zero at infinity if and only if

$$
\int^{\infty} \frac{\epsilon(y)}{y} d y<\infty
$$

In a similar direction the Paley-Wiener theorem 50 gives a characterization of a function or distribution with compact support in term of analyticity properties of its Fourier transform.

Regarding our results discussed above it would be interesting to identify a class of potentials $V(x, t)$ for which a result of the following kind holds:

If $u \in C\left([0,1]: L^{2}\left(\mathbb{R}^{n}\right)\right)$ is a nontrivial solution of the IVP

$$
\left\{\begin{array}{l}
\partial_{t} u=i(\triangle u+V(x, t) u), \quad(x, t) \in \mathbb{R}^{n} \times[0,1], \\
u(x, 0)=u_{0}(x),
\end{array}\right.
$$

with $u_{0} \in L^{2}\left(\mathbb{R}^{n}\right)$ having compact support, then $e^{\epsilon|x|} u(\cdot, t) \notin$ $L^{2}\left(\mathbb{R}^{n}\right)$ for any $\epsilon>0$ and any $t \in(0,1]$.

In this direction we have the following result which will appear in [40]:

Theorem 10. Assume that $u \in C\left([0,1]: L^{2}\left(\mathbb{R}^{n}\right)\right)$ is a strong solution of the IVP (2.4) with

$$
\begin{aligned}
& \operatorname{supp} u_{0} \subset B_{R}(0)=\left\{x \in \mathbb{R}^{n}:|x| \leq R\right\}, \\
& \int_{\mathbb{R}^{n}} e^{2 a_{1}|x|}|u(x, 1)|^{2} d x<\infty, \quad a_{1}>0,
\end{aligned}
$$

and

$$
\|V\|_{L^{\infty}\left(\mathbb{R}^{n} \times[0,1]\right)}=M_{0},
$$

with

$$
\lim _{R \rightarrow+\infty}\|V\|_{L^{1}\left([0,1]: L^{\infty}\left(\mathbb{R}^{n} \backslash B_{R}\right)\right)}=0 .
$$

Then, there exists $b=b(n)>0$ (depending only on the dimension $n$ ) such that if

$$
\frac{a_{1}}{R\left(M_{0}+1\right)} \geq b
$$

then $u \equiv 0$.

A similar question can be raised for results of the type described above due to A. E. Ingham in 32 and possible extensions to higher dimensions $n \geq 2$. 
It would be interesting to obtain extensions of the above results characterizing the decay of the solution $u(x, t)$ to the equation (1.6) with $F$ as in (1.8) associated to data $u_{0} \in L^{2}\left(\mathbb{R}^{n}\right)$ with compact support or with $u_{0} \in C_{0}^{\infty}\left(\mathbb{R}^{n}\right)$. In this direction, some results can be deduced as a consequence of Theorem 10, see 40].

\section{HARDY'S UNCERTAINTY PRINCIPLE IN A HALF-SPACE}

In the introduction we have briefly reviewed some uniqueness results established for solutions of the Schrödinger equation vanishing at two different times in a semispace of $\mathbb{R}^{n}$ (see [56], 15], 33], [34, 20]). In section 2, we have studied uniqueness results obtained under the hypothesis that the solution of the Schrödinger equation at two different times has an appropriate Gaussian decay in the whole space $\mathbb{R}^{n}$. In this section, we shall deduce a unified result, i.e., a uniqueness result under the hypothesis that at two different times the solution of the Schrödinger equation has Gaussian decay in just a semispace of $\mathbb{R}^{n}$.

Theorem 11. Assume that $u \in C\left([0,1]: L^{2}\left((0, \infty) \times \mathbb{R}^{n-1}\right)\right)$ is a strong solution of the IVP

$$
\left\{\begin{array}{l}
\partial_{t} u=i(\Delta+V(x, t)) u, \\
u(x, 0)=u_{0}(x),
\end{array}\right.
$$

with

$$
\begin{gathered}
\int_{0}^{1} \int_{1 / 2}^{3 / 2}\left|\partial_{x_{1}} u(x, t)\right|^{2} d x d t<\infty, \\
V: \mathbb{R}^{n} \times[0,1] \rightarrow \mathbb{C}, \quad V \in L^{\infty}\left(\mathbb{R}^{n} \times[0,1]\right),
\end{gathered}
$$

and

$$
\lim _{R \rightarrow+\infty} \int_{0}^{1}\|V(t)\|_{L^{\infty}\left(\left\{x_{1}>R\right\}\right)} d t=0
$$

Assume that

$$
\int_{x_{1}>0} e^{c_{0}\left|x_{1}\right|^{2}}|u(x, 0)|^{2} d x<\infty
$$

$$
\int_{x_{1}>0} e^{c_{1}\left|x_{1}\right|^{2}}|u(x, 1)|^{2} d x<\infty
$$

with $c_{0}, c_{1}>0$ sufficiently large. Then $u \equiv 0$.

Remarks. (a) Note that in Theorem 11] the solution does not need to be defined for $x_{1} \leq 0$. In this sense, this is a stronger result that the uniqueness results in 56, [39], 33], 34], and [15], which required that the solution be defined in $\mathbb{R}^{n} \times[0,1]$ and be $C\left([0,1]: L^{2}\left(\mathbb{R}^{n}\right)\right)$.

On the other hand, we need to assume the condition (5.2). Note that [39] also needs an extra assumption on $\nabla u$, stronger that (5.2), but that in [33, which among other things removed any extra assumption on $\nabla u$, but still required the solution to be defined in $\mathbb{R}^{n} \times[0,1]$ and be in $C\left([0,1]: L^{2}\left(\mathbb{R}^{n}\right)\right)$. If, in the setting of Theorem 11, we know that $u$ is a solution in $\mathbb{R}^{n} \times[0,1]$ and is in $C\left([0,1]: L^{2}\left(\mathbb{R}^{n}\right)\right)$, then we can dispose the hypothesis (5.2) as follows.

First, as in the first step of the proof of Theorem 11, we can use the Appell transformation to reduce to the case $c_{1}=c_{2}=2 \gamma$. Then, using $\varphi\left(x_{1}\right)$ a regularized 
convex function which agrees with $x_{1}^{+}$for $x_{1}>1, x_{1}<-1$, an application of Lemma 3 and Corollary 3 in the appendix yields the estimate

$$
\sup _{0 \leq t \leq 1} \int e^{2 \gamma\left(x_{1}^{+}\right)^{2}}|u(x, t)|^{2} d x+\int_{0}^{1} \int_{x_{1}>2} t(1-t)|\nabla u(x, t)|^{2} e^{2 \gamma\left(x_{1}^{+}\right)^{2}} d x d t<\infty .
$$

Once this is obtained, by restricting our attention to

$$
(2, \infty) \times \mathbb{R}^{n-1} \times[\delta, 1-\delta],
$$

for each $\delta>0$, we are in the situation of Theorem [1] and hence $u \equiv 0$ on $\left\{x_{1}>2\right\} \times[0,1]$. Finally, Izakov's result in 35] concludes that $u \equiv 0$ (more precisely, the version of Izakov's result proved in [33], which does not require $\nabla u$ to exist for $-1<x_{1}<1$ ).

(b) We have seen that Theorem 11 includes many of the uniqueness results for solutions vanishing at two different times in a semispace. In comparison with the results in section 2 , since the extra assumption (5.2) can be recovered as in Remark (a) when the solution is defined in $\mathbb{R}^{n} \times[0,1]$ and is in $C\left([0,1]: L^{2}\left(\mathbb{R}^{n}\right)\right)$, the only weakness is that they provide an optimal estimate for the constants $c_{1}, c_{2}$, but on the other hand, they deal with solutions only defined in $(0, \infty) \times \mathbb{R}^{n-1} \times[0,1]$.

(c) In Theorem 11] the direction $\vec{e}_{1}$ can be replaced by any other $\omega \in \mathcal{S}^{n-1}$.

Proof of Theorem 11. The strategy of the proof follows closely the one in [16]. We divide the proof into three steps.

Step 1 . Reduction to the case $c_{0}=c_{1}=2 \gamma$.

This follows by using the conformal or Appell transformation introduced in section 2 (see (2.7)-(2.11) ), combined with the observation that the set $\left\{x_{1}>0\right\}$ remains invariant.

Step 2. Upper bounds.

We define

$$
v(x, t)=\theta\left(x_{1}\right) u(x, t),
$$

with $\theta \in C^{\infty}(\mathbb{R})$, nondecreasing with $\theta\left(x_{1}\right) \equiv 1$ if $x_{1}>3 / 2$, and $\theta\left(x_{1}\right) \equiv 0$ if $x_{1}<1 / 2$. Therefore,

$$
\partial_{t} v=i \Delta v+i V(x, t) v+i F(x, t), \quad F(x, t)=2 \partial_{x_{1}} u \theta^{\prime}\left(x_{1}\right)+u \theta^{\prime \prime}\left(x_{1}\right) .
$$

Using (5.2), we can apply Lemma 1 to get that

$$
\begin{aligned}
& \sup _{0 \leq t \leq 1}\left\|e^{\lambda \cdot x_{1}} v(\cdot, t)\right\|_{L^{2}\left(\mathbb{R}^{n}\right)} \\
& \leq c_{n}\left(\left\|e^{\lambda \cdot x_{1}} v(0)\right\|_{L^{2}\left(\mathbb{R}^{n}\right)}+\left\|e^{\lambda \cdot x_{1}} v(1)\right\|_{L^{2}\left(\mathbb{R}^{n}\right)}\right. \\
& \left.+\int_{0}^{1}\left\|e^{\lambda \cdot x_{1}} F(\cdot, t)\right\|_{L^{2}\left(\mathbb{R}^{n}\right)} d t+\int_{0}^{1}\left\|e^{\lambda \cdot x_{1}} V \chi_{\left\{x_{1}<R\right\}} v(\cdot, t)\right\|_{L^{2}\left(\mathbb{R}^{n}\right)} d t\right),
\end{aligned}
$$

for some fixed $R$ sufficiently large. Thus, using (5.2)

$$
\begin{aligned}
& \sup _{0 \leq t \leq 1}\left\|e^{\lambda \cdot x_{1}} v(\cdot, t)\right\|_{L^{2}\left(\mathbb{R}^{n}\right)} \\
& \leq c_{n}\left(\left\|e^{\lambda \cdot x_{1}} v(0)\right\|_{L^{2}\left(\mathbb{R}^{n}\right)}+\left\|e^{\lambda \cdot x_{1}} v(1)\right\|_{L^{2}\left(\mathbb{R}^{n}\right)}\right. \\
& \left.+c e^{c|\lambda|}+c\|V\|_{\infty} e^{c|\lambda| R}\right) .
\end{aligned}
$$


Thus, from the formula (1.16) (with $p=2$ and $n=1$ ) and (5.8) we obtain that

$$
\begin{aligned}
& \sup _{0 \leq t \leq 1}\left\|e^{\gamma\left|x_{1}\right|^{2}} v(\cdot, t)\right\|_{L^{2}\left(\mathbb{R}^{n}\right)} \\
& \quad \leq\left(\left\|e^{\gamma\left|x_{1}\right|^{2}} v(0)\right\|_{L^{2}\left(\mathbb{R}^{n}\right)}+\left\|e^{\gamma\left|x_{1}\right|^{2}} v(1)\right\|_{L^{2}\left(\mathbb{R}^{n}\right)}+c+\|V\|_{\infty} e^{c \gamma R^{2}}\right) .
\end{aligned}
$$

Thus,

$$
\sup _{0 \leq t \leq 1}\left\|e^{\gamma\left|x_{1}\right|^{2}} v(\cdot, t)\right\|_{L^{2}\left(\mathbb{R}^{n}\right)} \leq c_{\gamma}
$$

Combining this and the equation for $v$, we shall get a smoothing estimate. Using the notation

$$
H(t)=\|f\|_{L^{2}\left(\mathbb{R}^{n}\right)}^{2}=\|f\|^{2},
$$

with

$$
f(x, t)=e^{\gamma\left|x_{1}\right|^{2}} v(x, t)
$$

and the abstract Lemma 3 (see the appendix), one formally has that

$$
\begin{aligned}
\partial_{t}^{2} H & \leq 2 \partial_{t} \operatorname{Re}\left(\partial_{t} f-\mathcal{S} f-\mathcal{A} f, f\right) \\
& +2\left(\mathcal{S}_{t} f+[\mathcal{S}, \mathcal{A}] f, f\right)+\left\|e^{\gamma\left|x_{1}\right|^{2}}(F+V v)\right\|^{2},
\end{aligned}
$$

with

$$
e^{\gamma\left|x_{1}\right|^{2}}\left(\partial_{t}-i \Delta\right)\left(e^{-\gamma\left|x_{1}\right|^{2}} f\right)=\partial_{t} f-\mathcal{S} f-\mathcal{A} f=e^{\gamma\left|x_{1}\right|^{2}}(F+V v),
$$

where $\mathcal{S}=-i \gamma\left(4 x_{1} \partial_{x_{1}}+2\right)$ is symmetric, $\mathcal{A}=i\left(\Delta+4 \gamma x_{1}^{2}\right)$ is skew-symmetric, and $F$ is as in (5.6). Since

$$
[\mathcal{S} ; \mathcal{A}]=-8 \gamma \partial_{x_{1}}^{2}+16 \gamma^{2} x_{1}^{2}
$$

using the inequality

$$
\begin{aligned}
& \int_{\mathbb{R}^{n}}\left(\left|\partial_{x_{1}} f\right|^{2}+4 \gamma^{2}\left|x_{1}\right|^{2}|f|^{2}\right) d x=\int_{\mathbb{R}^{n}} e^{2 \gamma\left|x_{1}\right|^{2}}\left(\left|\partial_{x_{1}} u\right|^{2}-2 \gamma|u|^{2}\right) d x \\
& \geq 2 \gamma \int_{\mathbb{R}^{n}}|f|^{2} d x
\end{aligned}
$$

together with Corollary 3 we conclude that

$$
\int_{0}^{1} \int t(1-t)\left|\partial_{x_{1}} v(x, t)\right|^{2} e^{2 \gamma\left|x_{1}\right|^{2}} e^{2 \gamma\left|x_{1}\right|^{2}} d x d t \leq c_{\gamma}
$$

Combining and (5.9) and (5.11) one gets that

$$
\begin{aligned}
& \sup _{0 \leq t \leq 1}\left\|e^{\gamma\left|x_{1}\right|^{2}} v(\cdot, t)\right\|_{L^{2}\left(\mathbb{R}^{n}\right)} \\
& +\int_{0}^{1} \int t(1-t)\left|\partial_{x_{1}} v(x, t)\right|^{2} e^{2 \gamma\left|x_{1}\right|^{2}} e^{2 \gamma\left|x_{1}\right|^{2}} \mid d x d t \leq c_{\gamma} .
\end{aligned}
$$

Step 3. We recall the following result, which is a slight variation of that proven in detail in [16] (Lemma 3.1, page 1818):

Lemma 2. Assume that $R>0$ and $\varphi:[0,1] \rightarrow \mathbb{R}$ is a smooth function. Then, there exists $c=c\left(n ;\left\|\varphi^{\prime}\right\|_{\infty}+\left\|\varphi^{\prime \prime}\right\|_{\infty}\right)>0$ such that the inequality

$$
\frac{\alpha^{3 / 2}}{R^{2}}\left\|e^{\alpha\left|\frac{x_{1}-x_{0}}{R}+\varphi(t)\right|^{2}} g\right\|_{L^{2}(d x d t)} \leq c\left\|e^{\alpha\left|\frac{x_{1}-x_{0}}{R}+\varphi(t)\right|^{2}}\left(i \partial_{t}+\Delta\right) g\right\|_{L^{2}(d x d t)}
$$


holds when $\alpha>c R^{2}$ and $g \in C_{0}^{\infty}\left(\mathbb{R}^{n+1}\right)$ is supported in the set

$$
\left\{(x, t)=\left(x_{1}, \ldots, x_{n}, t\right) \in \mathbb{R}^{n+1}:\left|\frac{x_{1}-x_{0_{1}}}{R}+\varphi(t)\right| \geq 1\right\} .
$$

Now, we will choose $x_{0_{1}}=R / 2, \quad 0 \leq \varphi(t) \leq a$, with $a=3 / 2-1 / R, \varphi(t)=a$, on $3 / 8 \leq t \leq 5 / 8, \varphi(t)=0$, for $t \in[0,1 / 4] \cup[3 / 4,1]$, and $\theta_{R} \in C^{\infty}(\mathbb{R})$ with $\theta_{R}\left(x_{1}\right)=1$ on $1<x_{1}<R-1$, and $\theta_{R}\left(x_{1}\right)=0$ for $x_{1}<1 / 2$ or $x_{1}>R$.

Also we choose $\eta \in C^{\infty}(\mathbb{R})$ with $\eta\left(x_{1}\right)=0, x_{1} \leq 1$ and $\eta\left(x_{1}\right)=1, x_{1} \geq$ $1+1 / 2 R$.

We notice that, up to translation, we can assume that

$$
\int_{3 / 8}^{5 / 8} \int_{2<x_{1}<3}|u(x, t)|^{2} d x d t=b \neq 0,
$$

otherwise we would have

$$
u(x, t)=0 \quad \text { on } \quad(x, t) \text { s.t. }\left(x_{1}, t\right) \in(0, \infty) \times(3 / 8,5 / 8),
$$

and thus by Izakov's result [35] we would get that $u \equiv 0$.

We let

$$
g(x, t)=\theta_{R}\left(x_{1}\right) \eta\left(\frac{x_{1}-R / 2}{R}+\varphi(t)\right) u(x, t) .
$$

It is easy to see that $g$ is supported on the set

$$
\left\{(x, t) \in \mathbb{R}^{n+1}: 1 / 2<x_{1}<R, 1 / 32<t<31 / 32,\left|\frac{x_{1}-R / 2}{R}+\varphi(t)\right| \geq 1\right\},
$$

and so satisfies the hypothesis of Lemma 2, Also if $\left(x_{1}, t\right) \in(2,3) \times(3 / 8,5 / 8)$, one has $\varphi=a, \eta\left(\frac{x_{1}-R / 2}{R}+a\right)=1$ and $\theta_{R}=1$, hence in this domain

$$
g(x, t)=u(x, t) .
$$

Thus, from (5.16) it follows that

$$
\left|\frac{x_{1}-R / 2}{R}+\varphi(t)\right| \geq 1+1 / R,
$$

so we have the lower bound of (5.13)

$$
\frac{\alpha^{3 / 2}}{R^{2}} b e^{\alpha(1+1 / R)^{2}}
$$

with $b$ as in (5.14). Now we shall estimate the right-hand side of (5.13). Thus,

$$
\begin{aligned}
& \left(i \partial_{t}-\Delta\right) g=-\theta_{R}\left(x_{1}\right) \eta\left(\frac{x_{1}-R / 2}{R}+\varphi(t)\right) V(x, t) u(x, t) \\
& \quad+\eta\left(\frac{x_{1}-R / 2}{R}+\varphi(t)\right)\left(2 \theta^{\prime}\left(x_{1}\right) \partial_{x_{1}} u+u \theta_{R}^{\prime \prime}\left(x_{1}\right)\right) \\
& \quad+\left(i \eta^{\prime}(\cdot) \varphi^{\prime}(t)+\eta^{\prime \prime}(\cdot) \frac{1}{R^{2}}\right) \theta_{R}\left(x_{1}\right) u(x, t) \equiv E_{1}+E_{2}+E_{3} .
\end{aligned}
$$

Choosing $R \gg\|V\|_{\infty}$, and recalling the fact that $\alpha>c R^{2}$, we see that the contribution of the term $E_{1}$ involving the potential $V$ can be absorbed by the term in the left-hand side of (5.13).

Next, we notice that the terms in $E_{2}$ involve derivatives of $\theta_{R}\left(\theta_{R}^{\prime}\right.$ or $\left.\theta_{R}^{\prime \prime}\right)$ so they are supported in the $(x, t) \in \mathbb{R}^{n} \times[0,1]$ such that

$$
1 / 2<x_{1}<1, \quad \text { or } R-1<x_{1}<R \text {. }
$$


But, if $1 / 2<x_{1}<1$, it follows that

$$
\frac{x_{1}-R / 2}{R}+\varphi(t) \leq 1 / R-1 / 2+3 / 2-1 / R=1, \quad \text { so } \eta\left(\frac{x_{1}-R / 2}{R}+\varphi(t)\right)=0 .
$$

Thus, we only get contribution from the $(x, t) \in \mathbb{R}^{n} \times[0,1]$ such that $R-1<x_{1}<R$, which can be bounded by

$$
c \int_{1 / 32}^{31 / 32} \int_{R-1<x_{1}<R}\left(|u|^{2}+\left|\partial_{x_{1}} u\right|^{2}\right)(x, t) e^{\alpha(2-1 / R)^{2}} d x d t .
$$

Finally, we look at the contribution of the term in $E_{3}$ in (5.17). In those the derivatives fall on $\eta$, thus they are supported in the region

$$
1 \leq \frac{x_{1}-R / 2}{R}+\varphi(t) \leq 1+\frac{1}{2 R}, \quad \frac{1}{2}<x_{1}<R, \quad \frac{1}{32}<t<\frac{31}{32} .
$$

Hence, their contribution in (5.13) is bounded by

$$
c \int_{1 / 32}^{31 / 32} \int_{1 / 2<x_{1}<R}|u(x, t)|^{2} e^{\alpha(1+1 /(2 R))^{2}} d x d t \leq c_{\gamma} e^{\alpha(1+1 /(2 R))^{2}} .
$$

Defining

$$
\delta(R)=\int_{1 / 32}^{31 / 32} \int_{R-1<x_{1}<R}\left(|u|^{2}+\left|\partial_{x_{1}} u\right|^{2}\right)(x, t) d x d t
$$

and collecting the above information using that $\alpha=c_{n} R^{2}$, we get

$$
c R b e^{\alpha(1+1 / R)^{2}} \leq c \delta(R) e^{\alpha(2-1 / R)^{2}}+c_{\gamma} e^{\alpha(1+1 /(2 R))^{2}} .
$$

Therefore, for $R$ sufficiently large it follows that (since $b \neq 0$ )

$$
c R b e^{\alpha(1+1 / R)^{2}} \leq c \delta(R) e^{\alpha(2-1 / R)^{2}},
$$

and since $\alpha=c_{n} R^{2}$ one has that

$$
\delta(R) \geq b e^{-c_{n} R^{2}} .
$$

To conclude, we recall that the upper bounds in (5.12) gave us

$$
\delta(R) \leq c e^{-\gamma R^{2}},
$$

hence if $\gamma>c_{n} / 2$, we conclude that $b=0$, which yields the desired result $u \equiv 0$.

\section{Appendix}

Above we have used the following abstract results established in 20]:

Lemma 3. Let $\mathcal{S}$ be a symmetric operator, let $\mathcal{A}$ be a skew-symmetric one, and let both be allowed to depend on the time variable. Let $G$ be a positive function, let $f(x, t)$ be a reasonable function, and

$$
\begin{aligned}
& H(t)=(f, f)=\|f\|_{L^{2}\left(\mathbb{R}^{n}\right)}^{2}=\|f\|^{2}, \quad D(t)=(\mathcal{S} f, f), \\
& \partial_{t} \mathcal{S}=\mathcal{S}_{t} \quad \text { and } \quad N(t)=\frac{D(t)}{H(t)} .
\end{aligned}
$$

Then

$$
\begin{aligned}
\partial_{t}^{2} H & =2 \partial_{t} \operatorname{Re}\left(\partial_{t} f-\mathcal{S} f-\mathcal{A} f, f\right)+2\left(\mathcal{S}_{t} f+[\mathcal{S}, \mathcal{A}] f, f\right) \\
& +\left\|\partial_{t} f-\mathcal{A} f+\mathcal{S} f\right\|^{2}-\left\|\partial_{t} f-\mathcal{A} f-\mathcal{S} f\right\|^{2}
\end{aligned}
$$


and

$$
\dot{N}(t) \geq\left(\mathcal{S}_{t} f+[\mathcal{S}, \mathcal{A}] f, f\right) / H-\left\|\partial_{t} f-\mathcal{A} f-\mathcal{S} f\right\|^{2} /(2 H)
$$

Moreover, if

$$
\left|\partial_{t} f-\mathcal{A} f-\mathcal{S} f\right| \leq M_{1}|f|+G, \text { in } \mathbb{R}^{n} \times[0,1], \quad \mathcal{S}_{t}+[\mathcal{S}, \mathcal{A}] \geq-M_{0},
$$

and

$$
M_{2}=\sup _{[0,1]}\|G(t)\| /\|f(t)\|
$$

is finite, then $\log H(t)$ is logarithmically convex in $[0,1]$ and there is a universal constant $N$ such that

$$
H(t) \leq e^{N\left(M_{0}+M_{1}+M_{2}+M_{1}^{2}+M_{2}^{2}\right)} H(0)^{1-t} H(1)^{t} \text {, when } 0 \leq t \leq 1 .
$$

By multiplying the formula (6.1) by $t(1-t)$, integrating the result over $[0,1]$, and using integration by parts, one gets the following smoothing inequality:

Corollary 3. With the same hypotheses and notation as in Lemma 3

$$
\begin{aligned}
& 2 \int_{0}^{1} t(1-t)\left(\mathcal{S}_{t} f+[\mathcal{S}, \mathcal{A}] f, f\right) d t+\int_{0}^{1} H(t) d t \leq H(0)+H(1) \\
& +2 \int_{0}^{1}(1-2 t) \operatorname{Re}\left(\partial_{t} f-\mathcal{S} f-\mathcal{A} f, f\right) d t \\
& +\int_{0}^{1} t(1-t)\left\|\partial_{t} f-\mathcal{A} f-\mathcal{S} f\right\|_{2}^{2} d t
\end{aligned}
$$

\section{ABOut THE AUthors}

Luis Escauriaza is a professor in the Department of Mathematics at the University of the Basque Country in Bilbao, Spain.

Carlos E. Kenig is the Louis Block Distinguished Service Professor in Mathematics and the College at the University of Chicago. He has been awarded the Salem Prize (1984) and the AMS Bocher Prize (2008), and he was a plenary speaker at the International Congress of Mathematicians in Hyderabad in 2010.

Gustavo Ponce is a professor of mathematics at the University of California, Santa Barbara. He was an invited speaker at the International Congress of Mathematicians in Berlin in 1998.

Luis Vega is a professor in the Department of Mathematics at the University of the Basque Country in Bilbao, Spain. He was an invited speaker at the International Congress of Mathematicians in Madrid in 2006.

\section{References}

[1] N. Aronszajn, A. Krzywicki, and J. Szarski, Unique continuation theorem for exterior differential forms on Riemannian manifolds, Ark. Math. 4 (1962) 417-453. MR0140031(25:3455)

[2] S. Ben Farah, and K. Mokni, Uncertainty principles and the $L^{p}-L^{q}$-version of Morgan's theorem on some groups, Russian J. Math. Physics 10 (2003) 245-260. MR2012899 (2004h:43010)

[3] H. Berestycki, and P.-L. Lions, Nonlinear scalar field equations, Arch. Rational Mech. Anal. 82 (1983) 313-375. MR695535 (84h:35054a)

[4] H. Berestycki, T. Gallouët, and O. Kavian, Équations de champs scalaires Euclidiens non linéaires dans de plan, C. R. Acad. Sci. Paris, Ser. I Math. 297 (1983) 307-310. MR734575 (85e:35041)

[5] A. Bonami, and B. Demange, A survey on uncertainty principles related to quadratic forms, Collect. Math. Vol. Extra (2006) 1-36. MR2264204 (2007g:42018) 
[6] A. Bonami, B. Demange, and P. Jaming, Hermite functions and uncertainty principles for the Fourier and the windowed Fourier transforms, Rev. Mat. Iberoamericana 19 (2006) 23-55. MR.1993414 (2004f:42015)

[7] J. Bourgain, and C. E. Kenig, On localization in the continuous Anderson-Bernoulli model in higher dimensions, Invent. Math. 161, 2 (2005) 1432-1297. MR2180453 (2006k:82085)

[8] A. P. Calderón, Uniqueness in the Cauchy problem for partial differential equations, Amer. J. Math., 80 (1958), 16-36. MR0104925 (21:3675)

[9] T. Carleman, Sur un probléme d'unicité pour les systémes d' equations aux derivées partielles á deux variables indépendantes, Ark. Math. 26B, (1939) 1-9.

[10] S. Chanillo, Uniqueness of solutions to Schrödinger equations on complex semi-simple Lie groups, Proc. Indian Acad. Sci. Math. Sci. 117 (2007), 325-331. MR2352052 (2008h:22010)

[11] M. Cowling, L. Escauriaza, C. E. Kenig, G. Ponce, and L. Vega, The Hardy uncertainty principle revisited, to appear in Indiana U. Math. J.

[12] M. Cowling, and J. F. Price, Generalizations of Heisenberg's inequality, Harmonic Analysis (Cortona, 1982) Lecture Notes in Math., 992 (1983), 443-449, Springer, Berlin. MR729369 (86g:42002b)

[13] M. Cowling, and J. F. Price, Bandwidth versus time concentration: the Heisenberg-PauliWeyl inequality, SIAM J. Math. Anal. 15 (1984) 151-165. MR728691 (86g:42002a)

[14] J. Cruz-Sanpedro, Unique continuation at infinity of solutions to Schrödinger equations with complex potentials, Proc. Roy. Soc. Edinburgh. 42 (1999) 143-153. MR1669361 (99m:35038)

[15] H. Dong, and W. Staubach. Unique continuation for the Schrödinger equation with gradient vector potentials, Proc. AMS 135, 7 (2007) 2141-2149. MR2299492 (2008e:35160)

[16] L. Escauriaza, C. E. Kenig, G. Ponce, and L. Vega, On uniqueness properties of solutions of Schrödinger equations, Comm. PDE. 31, 12 (2006) 1811-1823. MR2273975 (2009a:35198)

[17] L. Escauriaza, C. E. Kenig, G. Ponce, and L. Vega, On uniqueness properties of solutions of the k-generalized KdV, J. of Funct. Anal. 244, 2 (2007) 504-535. MR2297033 (2007k:35406)

[18] L. Escauriaza, C. E. Kenig, G. Ponce, L. Vega, Decay at infinity of caloric functions within characteristic hyperplanes, Math. Res. Letters 13, 3 (2006) 441-453. MR2231129 (2007e:35125)

[19] L. Escauriaza, C. E. Kenig, G. Ponce, and L. Vega, Convexity of free solutions of Schrödinger equations with Gaussian decay, Math. Res. Lett. 15, 5 (2008) 957-971. MR2443994 (2010m:35412)

[20] L. Escauriaza, C. E. Kenig, G. Ponce, and L. Vega, Hardy's uncertainty principle, convexity and Schrödinger evolutions, J. European Math. Soc. 10, 4 (2008) 883-907. MR2443923 (2009g:35248)

[21] L. Escauriaza, C. E. Kenig, G. Ponce, and L. Vega, Uncertainty principle of Morgan type and Schrödinger evolution, J. London Math. Soc. 81, (2011) 187-207. MR2763951|(2012a:35262)

[22] L. Escauriaza, C. E. Kenig, G. Ponce, and L. Vega, The sharp Hardy uncertainty principle for Schrödinger evolutions, Duke Math. J. 155, (2010) 163-187. MR2730375

[23] L. Escauriaza, C. E. Kenig, G. Ponce, and L. Vega, Unique continuation for Schrödinger evolutions, with applications to profiles of concentration and traveling waves, Comm. Math. Phys. 305, (2011) 487-512. MR2805469

[24] L. Escauriaza, G. Seregin, and V. Śverák, $L^{3, \infty}$ solutions to the Navier-Stokes equations and backward uniqueness, Russ. Math. Surv. 58, (2003) 211-250. MR1992563 (2004m:35204)

[25] L. Escauriaza, and S. Vessella, Optimal three cylinder inequalities for solutions to parabolic equations with Lipschitz leading coefficients, Contemp. Math. 333, Inverse Problems Theory and Applications (2002) 79-87. MR2032008 (2004k:35163)

[26] I. M. Gel'fand, and G. E. Shilov, Fourier transforms of rapidly increasing functions and questions of uniqueness of the solution of Cauchy's problem, Uspehi Matem. Nauk 8, (1953), 3-54. MR0061694 (15:867e)

[27] J. Hadamard, Le problóme de Cauchy et les équations aux derivées partielles linéaires hyperboliques, Hermann, Paris (1932)

[28] G. H. Hardy, A theorem concerning Fourier transforms, J. London Math. Soc. s1-8 (1933) $227-231$.

[29] N. Hayashi, K. Nakamitsu, and M. Tsutsumi, On solutions of the initial value problem for the nonlinear Schrödinger equations in one space dimension, Math. Z. 192 (1986) 637-650. MR.847012 (88b:35175) 
[30] N. Hayashi, K. Nakamitsu, and M. Tsutsumi, On solutions of the initial value problem for the nonlinear Schrödinger equations, J. Funct. Anal. 71 (1987) 218-245. MR880978 (88e:35162)

[31] L. Hörmander, A uniqueness theorem of Beurling for Fourier transform pairs, Ark. Mat. 29, 2 (1991) 237-240. MR1150375 (93b:42016)

[32] A. E. Ingham, A note on Fourier transforms J. London Math. Soc. s1-9 (1934) 29-32.

[33] A. D. Ionescu, and C. E. Kenig, $L^{p}$-Carleman inequalities and uniqueness of solutions of nonlinear Schrödinger equations, Acta Math. 193, 2 (2004) 193-239. MR2134866(2005m:35283)

[34] A. D. Ionescu, and C. E. Kenig, Uniqueness properties of solutions of Schrödinger equations, J. Funct. Anal. 232 (2006) 90-136. MR2200168 (2007b:35300)

[35] V. Izakov Carleman type estimates in an anisotropic case and applications, J. Differential Eqs. 105 (1993) 217-238. MR 1240395 (94k:35070)

[36] C. E. Kenig, Some recent quantitative unique continuation theorem, Rendiconti Accademia Nazionale dell Scienze, vol. XXIX (2005) 231-242. MR2305071 (2007m:35030)

[37] C. E. Kenig, Some recent applications of unique continuations, Contemp. Math. 439 (2007) 25-56. MR.2359019 (2009a:35016)

[38] C. E. Kenig, and F. Merle, Global well-posedness scattering and blow-up for the energycritical focusing non-linear wave equation, Acta Math. 201 (2008) 147-212. MR2461508 (2011a:35344)

[39] C. E. Kenig, G. Ponce, and L. Vega, On unique continuation for nonlinear Schrödinger equations, Comm. Pure Appl. Math. 60 (2002) 1247-1262. MR.1980854 (2004g:35195)

[40] C. E. Kenig, G. Ponce, L. Vega, A theorem of Paley-Wiener type for Schrödinger evolutions, to appear.

[41] V. A. Kondratiev, and E. M. Landis, Qualitative properties of the solutions of a secondorder nonlinear equation, Encyclopedia of Math. Sci. 32 (Partial Differential equations III) Springer-Verlag, Berlin, 1988. MR973885

[42] K. M. Kwong, Uniqueness of positive solutions of $\Delta u-u+u=0$ in $\mathbb{R}^{n}$, Arch. Rational Mech. Anal. 105 (1989) 243-266. MR969899 (90d:35015)

[43] E. M. Landis, and O. A. Oleinik, Generalized analyticity and certain properties, of solutions of elliptic and parabolic equations, that are connected with it, Uspehi Mat. Nauk 29 (1974), 190-206. MR0402268 (53:6089)

[44] J. L. Lions, and B. Malgrange, Sur l'unicité rétrograde dans les problèmes mixtes paraboliques, Math. Scan. 8 (1960) 277-286. MR0140855 (25:4269)

[45] V. Z. Meshkov, On the possible rate of decay at infinity of solutions of second-order partial differential equations, Math. USSR Sbornik 72 (1992), 343-361. MR.1110071 (92d:35032)

[46] G. W. Morgan, A note on Fourier transforms, J. London Math. Soc. 9 (1934), 187-192.

[47] C. Müller, On the behavior of the solution of the differential equation $\Delta u=f(x, u)$ in the neighborhood of a point, Comm. Math. Pure Appl. 1 (1954), 505-515. MR0062920 (16:42c)

[48] E. K. Naranayan and S. K. Ray, Beurling's theorem in $\mathbb{R}^{n}$, preprint.

[49] T. Nguyen, On a question of Landis and Oleinik, Trans. Amer. Math. Soc. 362 (2010) 28752899 MR2592940 (2011b:35186)

[50] R. Paley and N. Wiener, Fourier transform in the complex domain, Amer. Math. Soc Providence RI (1934). MR1451142(98a:01023)

[51] J.-C. Saut and B. Scheurer, Unique continuation for some evolution equations, J. Differential Eqs. 66 (1987), 118-139. MR871574(88a:35115)

[52] G. Seregin, A certain necessary condition of potential blow-up for Navier-Stokes equations, preprint, arXiv: 1104.3615 MR 2749374

[53] A. Sitaram, M. Sundari, and S. Thangavelu, Uncertainty principles on certain Lie groups, Proc. Indian Acad. Sci. Math. Sci. 105 (1995), 135-151. MR1350473 (96h:43002)

[54] W. A. Strauss, Existence of solitary waves in higher dimensions, Comm. Math. Phys. 55 (1977), 149-162. MR0454365 (56:12616)

[55] S. Vessella, Three cylinder inequalities and unique continuation properties for parabolic equations, Atti Accad. Naz. Lincei Cl. Sci. Fis. Mat. Natur. Rend. Lincei (9) Mat. Appl. 13 (2002), no. 2, 107-120. MR.1949484 (2004j:35036)

[56] B. Y. Zhang, Unique continuation properties of the nonlinear Schrödinger equations, Proc. Roy. Soc. Edinburgh. 127 (1997) 191-205. MR1433092 (97j:35143) 
UPV/EHU, Depto. De Matemáticas, Apto. 644, 48080 Bilbao, Spain

E-mail address: luis.escauriaza@ehu.es

Department of Mathematics, University of Chicago, Chicago, Illinois 60637

E-mail address: cek@math.uchicago.edu

Department of Mathematics, University of California, Santa Barbara, California 93106

E-mail address: ponce@math.ucsb.edu

UPV/EHU, Depto. De Matemáticas, Apto. 644, 48080 Bilbao, Spain

E-mail address: luis.vega@ehu.es 